\title{
Mean field analysis of reverse annealing for code-division multiple-access multiuser detection
}

\author{
Shunta Arai $\odot,{ }^{1,2, *}$ Masayuki Ohzeki, ${ }^{1,2,3}$ and Kazuyuki Tanaka ${ }^{1}$ \\ ${ }^{1}$ Graduate School of Information Sciences, Tohoku University, Sendai 980-8579, Japan \\ ${ }^{2}$ Sigma-i Co., Ltd., Minato, Tokyo 108-0075, Japan \\ ${ }^{3}$ Institute of Innovative Research, Tokyo Institute of Technology, Nagatsuta-cho 4259, Midori-ku, Yokohama, Kanagawa 226-8503, Japan
}

(Received 23 April 2020; accepted 28 April 2021; published 2 July 2021)

\begin{abstract}
Code-division multiple-access (CDMA) multiuser detection is a kind of signal recovery problem. The main problem of CDMA multiuser detection is to estimate the original signal from the degraded information. In CDMA multiuser detection, the first-order phase transition happens. The first-order phase transition degrades the estimation performance. To avoid or mitigate the first-order phase transition, we apply adiabatic reverse annealing (ARA) to CDMA multiuser detection. In ARA, we introduce the initial Hamiltonian, which corresponds to the prior information of the original signal into quantum annealing (QA) formulation. The ground state of the initial Hamiltonian is the initial candidate solution. By using the prior information of the original signal, ARA enhances the performance of QA for CDMA multiuser detection. We evaluate the typical ARA performance of CDMA multiuser detection by means of statistical mechanics using the replica method. At first, we consider the oracle cases where the initial candidate solution is randomly generated with a fixed fraction of the original signal in the initial state. In the oracle cases, the first-order phase transition can be avoided or mitigated by ARA if we prepare for the proper initial candidate solution. We validate our theoretical analysis with quantum Monte Carlo simulations. The theoretical results to avoid the first-order phase transition are consistent with the numerical results. Next, we consider the practical cases where we prepare for the initial candidate solution obtained by commonly used algorithms. We show that the practical algorithms can exceed the threshold to avoid the first-order phase transition. Finally, we test the performance of ARA with the initial candidate solution obtained by the practical algorithm. In this case, ARA cannot avoid the first-order phase transition even if the initial candidate solution exceeds the threshold to avoid the first-order phase transition.
\end{abstract}

DOI: 10.1103/PhysRevResearch.3.033006

\section{INTRODUCTION}

Code-division multiple-access (CDMA) multiuser detection has been used in various communication systems [1]. The theoretical performance of CDMA multiuser detection has been analyzed by means of statistical mechanics [2-5]. CDMA multiuser detection is regarded as a type of signal recovery problem like compressed sensing [6-9]. Statisticalmechanical analyses for signal recovery problems focus on the inference of the original information from the degraded information with noise. The noise can be physically regarded as thermal fluctuations. By tuning the strength of the thermal fluctuations, the original signal can be estimated from the degraded one.

In addition to thermal fluctuations, quantum fluctuations may be used to estimate the signal. Several studies have demonstrated that quantum fluctuations such as the transverse field do not necessarily improve the performance of the inferences for image restoration, Sourlas codes, and CDMA

\footnotetext{
*shunta.arai.s6@dc.tohoku.ac.jp

Published by the American Physical Society under the terms of the Creative Commons Attribution 4.0 International license. Further distribution of this work must maintain attribution to the author(s) and the published article's title, journal citation, and DOI.
}

[10-12]. The optimal decoding performance with quantum fluctuations is inferior to that with thermal fluctuations in Bayes optimal cases. However, in certain non-Bayes optimal cases, for example, where a lower temperature than the true noise level is set, the decoding performance with finite quantum fluctuations and thermal fluctuations is superior to that with only thermal fluctuations. That implies the potential of the combination of quantum and thermal fluctuations for inference problems.

The performance of optimization algorithms with quantum fluctuations, which is known as quantum annealing (QA) [13-18] or adiabatic quantum computation [19,20], is equal to or better than that of an optimization algorithm with thermal fluctuations [21,22], which is known as simulated annealing (SA) [23]. The physical implementation of QA is realized by the quantum annealer [24-28]. The quantum annealer has been implemented in numerous applications, such as portfolio optimizations [29,30], traffic optimization [31], item listing for ecommerce [32], automated guided vehicles in factories [33], machine learning [34-38], quantum simulation [39-41], material design [42], and decoding algorithm [43].

In a closed system, QA begins from the ground state of the transverse field term, and the transverse field strength is gradually reduced. Following the Schrödinger equation, the trivial ground state evolves adiabatically into a nontrivial ground state of the target Hamiltonian, which corresponds to the 
solution of combinatorial optimization problems. The quantum adiabatic theorem guarantees a theoretically sufficient condition to obtain the ground state in QA [44]. The theorem indicates that the total computational time for obtaining the ground state is characterized by the minimum energy gap between the ground state and first excited state. The energy gap is related to the order of the phase transition. In the case of the first-order phase transition, the computational time for searching the ground state increases exponentially [45-48], which is the worst case of QA.

To avoid the first-order phase transition, many methods are proposed, for example, QA with a nonstoquastic Hamiltonian [49-52], inhomogeneous driving of the transverse field $[53,54]$, and reverse annealing (RA) $[55,56]$. RA is a protocol to restart the quantum dynamics starting from the resulting state of the standard procedure of QA. We expect that RA leads to a closer solution to the ground state as its output. To assess the performance of RA, we carefully classified its implementation into two methods: adiabatic reverse annealing (ARA) and iterated reverse annealing (IRA). The main difference between ARA and IRA is to incorporate the resulting state. One is to implement the resulting state by modification of the initial Hamiltonian, and the other introduces it as the initial condition.

In ARA, we modify the initial Hamiltonian according the resulting state. We assume that the resulting state is a candidate solution, which is sufficiently close to the ground state of the original problem we wish to solve. We prepare the initial Hamiltonian in ARA such that its ground state is the candidate solution. The procedure of ARA is outlined as follows: We start from the ground state of the initial Hamiltonian. Next, we gradually increase the effects of quantum fluctuations and search locally around the candidate solution. Thereafter, we gradually decrease the effects of quantum fluctuations. When the effects of quantum fluctuations disappear, the ground state or lower energy state of the original problem can be obtained. ARA is rather a theoretical approach for understanding the property of RA. Theoretical analysis has indeed shown that ARA can avoid the first-order phase transition for the $p$-spin model [57]. However, this protocol has not been implemented in the current quantum annealer.

The procedure of IRA is slightly different from ARA. The difference is that IRA starts from a classical state, which corresponds to the candidate solution without introducing the additional Hamiltonian. A similar protocol to IRA is feasible in the current quantum annealer. The performance of IRA can be analyzed from the dynamics, and it significantly depends on effect of heat bath. In a closed system, IRA has not enhanced the performance of QA [58]. In an open system, IRA has improved the performance of QA by incorporating the relaxation mechanisms [59].

In this paper, we focus on ARA because it dramatically enhances the performance of QA, and its performance can be analyzed by statistical mechanics. To the best of our knowledge, it remains unknown whether ARA is useful for certain practical problems. We apply ARA to CDMA multiuser detection, which is a representative example in signal recovery problems. The CDMA model is mainly characterized by the ratio of the number of users to that of the measurements, which is called the pattern ratio. In the low-temperature re- gions and the intermediate pattern ratio, the CDMA model has two solutions. This phenomenon reveals the existence of the first-order phase transition, which degrades the estimation efficacy. We use ARA to mitigate or avoid the estimation difficulty. In the ARA process, we set the initial Hamiltonian. The initial Hamiltonian is interpreted as prior information of the original signal in the context of the inference problems. We expect that the prior information of the original signal will mitigate the estimation difficulty

We consider the marginal posterior mode (MPM) estimation by ARA [60]. The estimated signal corresponds to the expectation of the signal over the Gibbs-Boltzmann distribution. The MPM estimation can be performed in the current quantum annealer, which provides samples from the density matrix incorporating both thermal and quantum fluctuations in $\sim 20 \mu$ s $[61,62]$. We analyze the average MPM estimation performance with ARA at a finite temperature using the replica method. The MPM estimation with ARA is regarded as the MPM estimation with quantum fluctuation incorporating the prior information of the original signal. The typical performance of the MPM estimation with quantum fluctuations for CDMA multiuser detection has been analyzed in a previous study [12]. The connection between this paper and the previous study is presented in Sec. II A.

In ARA, we need to prepare for the initial candidate solution. In the previous study $[57,58]$, how to prepare for the initial candidate solution was not considered. We investigate whether we can prepare for the proper initial candidate solution to avoid the first-order phase transition with commonly used algorithms. We test the performance of ARA with the initial candidate solution obtained by these practical algorithms. Although the implementation of ARA in the current quantum annealer has not yet been realized, our results provide a theoretical demonstration of ARA as a practical technique for signal recovery problems.

The remainder of this paper is organized as follows. In Sec. II, we review the previous study and present the formulation of the CDMA model with quantum fluctuations. In Sec. III, we extend the formulation for ARA. We derive the free energy under the replica symmetry (RS) ansatz and the static approximation. In Sec. IV, we illustrate the phase diagrams in ARA. At first, we consider oracle cases where the initial candidate solution is randomly generated from the probability distribution, given the fraction of the original signal in the initial state. To verify the RS solutions, we perform quantum Monte Carlo simulations. Next, we check whether we can prepare for the proper initial candidate solution to avoid the first-order phase transition with commonly used algorithms. Finally, we test the performance of ARA with the initial candidate solution attained from these practical algorithms. In Sec. V, we conclude the study and discuss the future research directions.

\section{CDMA MODEL WITH QUANTUM FLUCTUATIONS}

At first, we review the previous study [12] and show its relationship with the MPM estimation with ARA in Sec. II A. Next, we formulate the classical CDMA model and move onto the quantum system in Sec. II B. 


\section{A. Related Work}

The previous study [12] analyzed the performance of the MPM estimation for the CDMA model with quantum fluctuations under the standard protocol of QA. They shed light on the difference between quantum and thermal fluctuations. In other words, they compared the performance between SA and QA. In the case by SA, one controls the strength of thermal fluctuation through a parameter of temperature. Depending on the noise in the received signal, they found the optimal strength of the thermal fluctuation known as the Nishimori temperature [63] to retrieve the original signal in the context of CDMA. In the previous study, they investigated the existence of the optimal strength of the quantum fluctuation like the case with thermal fluctuation. They showed that the MPM estimation with quantum fluctuations could partially improve its performance compared with the case without quantum fluctuation. However, the MPM estimation with quantum fluctuations did not archive the optimal MPM performance found in the case only with thermal fluctuations. In this sense, the thermal fluctuation is superior to the quantum fluctuation in the retrieval of the original signal of CDMA. Nevertheless, one of the crucial bottlenecks of the protocol in both SA and QA to retrieve the original signal still exists. There is the firstorder phase transition in the case with the intermediate pattern ratio in the low-temperature region. Here, the temperature is a control parameter of the MPM estimation. The existence of the first-order phase transition hampers efficient retrieval of the original signal and needs the long computation time of its execution. As shown in the previous study, quantum fluctuation could not avoid nor mitigate the first-order phase transition. We thus investigate the potential of ARA, which is slightly different from the standard protocol of QA, in this paper. In this sense, this paper is placed in position as an extension of the MPM estimation with quantum fluctuations by using a different protocol of standard QA.

\section{B. Formulation}

The main concept of the CDMA model is as follows: The digital signal of each user is modulated and transmitted to a base station through fully synchronous channels. By demodulating the received signal composed of multiuser signals and noises, we infer the original signal from the provided information. The following formulation is mainly based on the previous study of the CDMA model with quantum fluctuations [12]. They add the transverse field to the original CDMA model and compute the partition function following the prescription of the statistical mechanics. They used the Suzuki-Trotter (ST) decomposition to deal with quantum fluctuation written in the transverse field and the replica method to compute the averaged free energy over the quenched randomness related to the signals and modulation. In this paper, we employ the same methods to tackle the MPM estimation of CDMA by using ARA and setting the initial Hamiltonian depending on the initial candidate solution.

We consider that $N$ users communicate via fully synchronous channels. At the base station, the receiver obtains the signal as follows:

$$
y^{\mu}=\frac{1}{\sqrt{N}} \sum_{i=1}^{N} \eta_{i}^{\mu} \xi_{i}+\epsilon^{\mu},
$$

where $\xi_{i} \in\{ \pm 1\},(i=1, \ldots, N)$ is the original information, and $\eta_{i}^{\mu} \in\{ \pm 1\}(i=1, \ldots, N, \mu=1, \ldots, K)$ is the spreading code for each user $i$. The length of the spreading codes for each user $i$ is represented by $K$. The channel noise $\epsilon^{\mu}$ is added into the received signal. The received signal in Eq. (1) can be expressed as

$$
\mathbf{y}=\frac{1}{\sqrt{N}} \eta \boldsymbol{\xi}+\boldsymbol{\epsilon},
$$

for which the following notations are used:

$$
\begin{gathered}
\mathbf{y}=\left(y^{1}, \ldots, y^{K}\right)^{T}, \quad \xi=\left(\xi_{1}, \ldots, \xi_{N}\right)^{T}, \\
\epsilon=\left(\epsilon^{1}, \ldots, \epsilon^{K}\right)^{T}, \\
\boldsymbol{\eta}=\left(\begin{array}{cccc}
\eta_{1}^{1} & \eta_{2}^{1} & \cdots & \eta_{N}^{1} \\
\eta_{1}^{2} & \eta_{2}^{2} & \cdots & \eta_{N}^{2} \\
\vdots & \vdots & \ddots & \vdots \\
\eta_{1}^{K} & \eta_{2}^{K} & \cdots & \eta_{N}^{K}
\end{array}\right) .
\end{gathered}
$$

We assume that the spreading codes and original signal are independently generated from the uniform distribution:

$$
\begin{gathered}
P(\eta)=\frac{1}{2^{N K}}, \\
P(\xi)=\frac{1}{2^{N}} .
\end{gathered}
$$

We consider the Gaussian channels, and $\epsilon^{k}$ is independently generated from the Gaussian distribution as follows:

$$
\begin{aligned}
P(\boldsymbol{\epsilon})=P(\mathbf{y} \mid \boldsymbol{\xi}) & =\left(\frac{1}{\sqrt{2 \pi T_{0}}}\right)^{K} \exp \left(-\frac{\|\boldsymbol{\epsilon}\|_{2}^{2}}{2 T_{0}}\right) \\
& =\left(\sqrt{\frac{\beta_{0}}{2 \pi}}\right)^{K} \exp \left(-\frac{\beta_{0}}{2}\left\|\mathbf{y}-\frac{\eta \xi}{\sqrt{N}}\right\|_{2}^{2}\right),
\end{aligned}
$$

where $T_{0}=\beta_{0}^{-1}$ is the true noise scale.

In CDMA multiuser detection, we estimate the original signal from the received output signal and the spreading codes that are prepared for each user in advance. Because the output signal fluctuates owing to noise, we formulate this problem as Bayesian inference. Subsequently, we introduce the posterior distribution of the estimated signal $\sigma=\left(\sigma_{1}, \ldots, \sigma_{N}\right)^{T}$ as

$$
P(\boldsymbol{\sigma} \mid \mathbf{y})=\frac{P(\mathbf{y} \mid \boldsymbol{\sigma}) P(\boldsymbol{\sigma})}{\operatorname{Tr} P(\mathbf{y} \mid \boldsymbol{\sigma}) P(\boldsymbol{\sigma})} .
$$

We define the likelihood as

$$
P(\mathbf{y} \mid \boldsymbol{\sigma})=\left(\sqrt{\frac{\beta}{2 \pi}}\right)^{K} \exp \left(-\frac{\beta}{2}\left\|\mathbf{y}-\frac{\eta \boldsymbol{\sigma}}{\sqrt{N}}\right\|_{2}^{2}\right)
$$

where $\beta=1 / T$ is the inverse temperature in statistical mechanics and corresponds to the estimated channel noise scale. If the true noise level is known, the estimation performance is the best and Bayes optimal. According to Eqs. (8) and (9), the posterior distribution can be written using the 
Gibbs-Boltzmann distribution with the Hamiltonian $H(\sigma)$, as follows:

$$
\begin{gathered}
P(\boldsymbol{\sigma} \mid \mathbf{y})=\frac{1}{Z} \exp \left\{-\beta\left[H(\boldsymbol{\sigma})+H_{\text {init }}(\boldsymbol{\sigma})\right]\right\}, \\
Z=\operatorname{Tr} \exp \left\{-\beta\left[H(\boldsymbol{\sigma})+H_{\text {init }}(\boldsymbol{\sigma})\right]\right\}, \\
H(\boldsymbol{\sigma})=\frac{1}{2 N} \sum_{i, j} \sum_{\mu=1}^{K} \eta_{i}^{\mu} \eta_{j}^{\mu} \sigma_{i} \sigma_{j}-\frac{1}{\sqrt{N}} \sum_{i=1}^{N} \sum_{\mu=1}^{K} \eta_{i}^{\mu} y^{\mu} \sigma_{i},
\end{gathered}
$$

where $Z$ is the partition function and $H_{\text {init }}(\sigma)$ is the initial Hamiltonian, which represents the prior information of the estimated signal. We generally assume that the prior of the estimated signal follows the uniform distribution

$$
P(\sigma)=\frac{1}{2^{N}} .
$$

In this case, we can omit the initial Hamiltonian from Eqs. (10) and (11).

To estimate the original signal, we consider the MPM estimation. The estimation performance can be evaluated by the overlap between the original and estimated signal as $\mathcal{M}=$ $1 / N \sum_{i=1}^{N} \xi_{i} \operatorname{sgn}\left\langle\sigma_{i}\right\rangle$, where $\langle\cdot\rangle$ is the expectation over the posterior distribution $P(\sigma \mid \mathbf{y})$ and $\operatorname{sgn}(\cdot)$ is the signum function. This quantity is expected to exhibit a "self-averaging" property in the thermodynamics limit $N \rightarrow \infty$. This means that the observables, such as the overlap for a quenched realization of the data $\mathbf{y}, \boldsymbol{\eta}$, and $\boldsymbol{\xi}$, are equivalent to the expectation of itself over the data distribution $P(\boldsymbol{\eta}) P(\boldsymbol{\xi}) P(\mathbf{y} \mid \boldsymbol{\xi})$. In this case, the overlap can be expressed as $\lim _{N \rightarrow \infty} \mathcal{M}=\left[\xi_{i} \operatorname{sgn}\left\langle\sigma_{i}\right\rangle\right]$, where the bracket $[\cdot]$ indicates the expectation over the data distribution.

It is straightforward to extend the above formulation into the quantum mechanical version:

$$
\begin{gathered}
\hat{H}=s \hat{H}_{0}+(1-s) \hat{H}_{\mathrm{TF}} \\
\hat{H}_{0}=\frac{1}{2 N} \sum_{i, j} \sum_{\mu=1}^{K} \eta_{i}^{\mu} \eta_{j}^{\mu} \hat{\sigma}_{i}^{z} \hat{\sigma}_{j}^{z}-\frac{1}{\sqrt{N}} \sum_{i=1}^{N} \sum_{\mu=1}^{K} \eta_{i}^{\mu} y^{\mu} \hat{\sigma}_{i}^{z} \\
\hat{H}_{\mathrm{TF}}=-\sum_{i=1}^{N} \hat{\sigma}_{i}^{x}
\end{gathered}
$$

where $\hat{\sigma}_{i}^{z}$ and $\hat{\sigma}_{i}^{x}$ are the $z$ and $x$ components of the Pauli matrices at site $i$, respectively. In this case, $\hat{H}_{0}$ consists of the $z$ components of the Pauli matrices, and $\hat{H}_{\mathrm{TF}}$ is composed of the $x$ components of the Pauli matrices. We parametrize the Hamiltonian in Eq. (14) with the annealing parameter $s$ for application to ARA.

As in the classical case, we consider the MPM estimation with quantum fluctuations. The posterior distribution can be written as $\hat{\rho}=\exp \{-\beta \hat{H}\} / \operatorname{Tr} \exp \{-\beta \hat{H}\}$, where $\operatorname{Tr}$ denotes the summation over all possible spin configurations in the $z$ basis. The performance of the MPM estimation with quantum fluctuations can be evaluated by $\mathcal{M}=$ $1 / N \sum_{i=1}^{N} \xi_{i} \operatorname{sgn}\left(\operatorname{Tr} \hat{\sigma}_{i}^{z} \hat{\rho}\right)$.

\section{MEAN FIELD ANALYSIS}

Following Ref. [57], we extend the CDMA model with quantum fluctuations [12] to the ARA formulation as

$$
\begin{gathered}
\hat{H}=s \hat{H}_{0}+(1-s)(1-\lambda) \hat{H}_{\mathrm{init}}+(1-s) \lambda \hat{H}_{\mathrm{TF}}, \\
\hat{H}_{\text {init }}=-\sum_{i=1}^{N} \tau_{i} \hat{\sigma}_{i}^{z},
\end{gathered}
$$

where $\lambda(0 \leqslant \lambda \leqslant 1)$ is the RA parameter. The initial candidate solution is denoted by $\tau_{i}= \pm 1(i=1, \ldots, N)$, which is expected to be close to the original signal $\xi_{i}$. We introduce the probability distribution of the initial candidate solution as follows:

$$
P(\boldsymbol{\tau})=\prod_{i=1}^{N} P\left(\tau_{i}\right)=\prod_{i=1}^{N}\left[c_{1} \delta\left(\tau_{i}-\xi_{i}\right)+c_{-1} \delta\left(\tau_{i}+\xi_{i}\right)\right],
$$

where we define $c_{1}=c$ and $c_{-1}=1-c$. The number $c(0 \leqslant$ $c \leqslant 1$ ) denotes the fraction of the original signal $\tau_{i}=\xi_{i}$ in the initial state as

$$
c=\frac{1}{N} \sum_{i=1}^{N} \delta_{\tau_{i}, \xi_{i}} .
$$

The prior information can be incorporated through Eq. (19). The main concept of the MPM estimation with ARA is to avoid or mitigate the first-order phase transition by controlling the RA parameter and utilizing the prior information.

The typical behaviors of the order parameters such as the overlap can be obtained via the free energy. We calculate the partition function $Z=\operatorname{Tr} \exp (-\beta \hat{H})$ and derive the $\mathrm{RS}$ free energy in the limit of $N, K \rightarrow \infty$, while maintaining the pattern ratio $\alpha \equiv K / N=O(1)$. The free energy density $f$ can be evaluated as $-\beta f=\lim _{N \rightarrow \infty}(1 / N)[\ln Z]$, where $[\cdot]$ denotes the configuration average over the data distribution $P(\mathbf{y} \mid \boldsymbol{\xi}) P(\boldsymbol{\eta}) P(\boldsymbol{\xi}) P(\boldsymbol{\tau})$. When computing $f$, we have two difficulties. The first one is the noncommutativity of the spin operator from Eq. (16). We cannot apply the mean-field analysis directly into the partition function of Eq. (17). The second one is to compute $[\ln Z]$. In general, it is difficult to directly evaluate $[\ln Z]$. We remove these two difficulties by using two techniques.

Firstly, to exclude the noncommutativity of the spin operator, we employ the ST decomposition [64] in the partition function:

$$
\begin{aligned}
Z & =\lim _{M \rightarrow \infty} \operatorname{Tr}\left\{\exp \left[-\frac{\beta}{M}\left(\hat{H}_{0}+\hat{H}_{\text {init }}\right)\right] \exp \left(-\frac{\beta}{M} \hat{H}_{\mathrm{TF}}\right)\right\}^{M} \\
& =\lim _{M \rightarrow \infty} Z_{M}
\end{aligned}
$$


where

$$
\begin{aligned}
Z_{M}= & \operatorname{Tr} \prod_{t=1}^{M}\left\{\frac{1}{2} \sinh \left[\frac{2 \beta(1-s) \lambda}{M}\right]\right\}^{\frac{N}{2}} \exp \left\{-\frac{\beta s}{2 N M} \sum_{i, j} \sum_{\mu=1}^{K} \eta_{i}^{\mu} \eta_{j}^{\mu} \sigma_{i}(t) \sigma_{j}(t)+\frac{\beta s}{M \sqrt{N}} \sum_{i=1}^{N} \sum_{\mu=1}^{K} \eta_{i}^{\mu} y^{\mu} \sigma_{i}(t)\right. \\
& \left.+\frac{\beta(1-s)(1-\lambda)}{M} \sum_{i=1}^{N} \tau_{i} \sigma_{i}(t)+\frac{1}{2} \ln \operatorname{coth}\left[\frac{\beta(1-s) \lambda}{M}\right] \sum_{i=1}^{N} \sigma_{i}(t) \sigma_{i}(t+1)\right\},
\end{aligned}
$$

in which the symbol $t$ is the index of the Trotter slice, and $M$ is the Trotter number. We impose the periodic boundary conditions, $\sigma_{i}(M+1)=\sigma_{i}(1)$ for all $i$. By using ST decomposition, we can map the quantum system into the identical classical system. The difficulty from the noncommutativity of the spin operator is removed. In the above expressions, we replace $\sigma_{i}^{z}(t)$ with the classical spin $\sigma_{i}(t) \in\{-1,+1\}$. In this case, the symbol Tr represents the trace over the classical spins. The $x$ component of the Pauli matrix yields the last term in Eq. (22).

Secondly, to evaluate $[\ln Z]$, we exploit the replica method [65]: $[\ln Z]=\lim _{n \rightarrow 0}\left(\left[Z^{n}\right]-1\right) / n$. The symbol $n$ denotes the number of replicas. By using the replica method, we can take the configuration average for the replicated partition function $Z^{n}$ and the limit of $n \rightarrow 0$. When manipulating the configuration average over $P(\mathbf{y} \mid \xi) P(\eta) P(\xi) P(\boldsymbol{\tau})$, we introduce the order parameters and their conjugate parameters through the $\delta$ function and its Fourier integral representation as follows: the magnetization $m_{a}(t)=(1 / N) \sum_{i=1}^{N} \xi_{i} \sigma_{i a}(t)$, the spin glass order parameter $q_{a b}\left(t, t^{\prime}\right)=(1 / N) \sum_{i=1}^{N} \sigma_{i a}(t) \sigma_{i b}\left(t^{\prime}\right)(a \neq b)$, and the correlation between each Trotter slice $R_{a}\left(t, t^{\prime}\right)=(1 / N) \sum_{i=1}^{N} \sigma_{i a}(t) \sigma_{i a}\left(t^{\prime}\right)$. The conjugate parameters are denoted by $\tilde{m}_{a}(t), \tilde{q}_{a b}\left(t, t^{\prime}\right)(a \neq b)$ and $\tilde{R}_{a}\left(t, t^{\prime}\right)$. These conjugate parameters appear in manipulation of several integrals over order parameters to compute the partition function as detailed in Appendix A. The symbols $a$ and $b$ represent the replica indices. Under the RS ansatz and static approximation $m_{a}(t)=m, q_{a b}\left(t, t^{\prime}\right)=q, R_{a}\left(t, t^{\prime}\right)=R, \tilde{m}_{a}(t)=\tilde{m}, \tilde{q}_{a b}\left(t, t^{\prime}\right)=\tilde{q}, \tilde{R}_{a}\left(t, t^{\prime}\right)=\tilde{R}$, we can finally obtain the RS free energy density:

$$
\begin{aligned}
-\beta f_{\mathrm{RS}}= & \underset{\substack{m, q, R \\
\tilde{m}, \tilde{q}, \tilde{R}}}{\operatorname{extr}}\left(\frac{\alpha}{2}\left\{-\ln [1+\beta s(R-q)]+\beta s\left[(R-1)+\frac{1+\beta_{0}}{\beta_{0}}+\frac{2 m-q-\left(1+\beta_{0}^{-1}\right)}{1+\beta s(R-q)}\right]\right\}\right. \\
& \left.-m \tilde{m}-R \tilde{R}+\frac{1}{2} q \tilde{q}+\sum_{a= \pm 1} c_{a} \int D z \ln \int D y 2 \cosh \sqrt{g_{a}^{2}+[\beta(1-s) \lambda]^{2}}\right),
\end{aligned}
$$

where

$$
g_{a}=\tilde{m}+a \beta(1-\lambda)(1-s)+\sqrt{\tilde{q}} z+\sqrt{2 \tilde{R}-\tilde{q}} y,
$$

in which $D z$ means that the Gaussian measure $D z:=d z / \sqrt{2 \pi} e^{-z^{2} / 2}$, and $D y$ is the same as $D z$. Here, extr represents the extremization by changing the order parameters $m, q$, and $R$ and their conjugate parameters as $\tilde{m}, \tilde{q}$, and $\tilde{R}$. The extremum point is determined by the saddle-point conditions and characterizes the free energy density. The expression in Eq. (23) for $\lambda=1$ can be reduced to the RS free energy density derived in Ref. [12]. The detailed derivation of Eq. (23) is written in Appendix A. The saddle-point equations are referred in Appendix B. Below, we investigate the phase transition of the order parameters while tuning the external parameters as the strength of the transverse field, the pattern ratio, etc. Then we numerically solve the saddle-point equations for each set of external parameters.

\section{NUMERICAL RESULTS}

In this section, we evaluate the typical performance of ARA based on the results attained in Sec. III. In Sec. IV A, we consider the oracle cases where the initial candidate solution is randomly generated from Eq. (19), given the fraction of the original signal in the initial state. In Sec. IV B, we consider the practical cases where we prepare for the initial candidate solution with commonly used algorithms. We compare the performance of ARA with the oracle cases and the practical cases.

\section{A. Analysis of ARA in oracle cases}

We numerically solve the saddle-point equations in Eqs. (B1) to (B6) with the temperature $T=0.1$. We set the several RA parameters $\lambda$. We start from ARA with $\lambda=1$, which corresponds to vanilla QA [12]. We show that the
CDMA model has the first-order phase transition in the intermediate pattern ratio. Next, we consider the classical case with $\lambda=0$ to validate the RS ansatz without the static approximation. Finally, we move onto the finite $\lambda$. We exhibit that ARA can avoid or mitigate the first-order phase transition if we prepare for the proper initial candidate solution.

\section{ARA with $\lambda=1$}

Let us begin with ARA with $\lambda=1$. The phase diagrams for the true noise scale $T_{0}=0,0.05$, and 0.1 are displayed in Fig. 1. The blue solid curve and orange dash-dotted curve indicate the spinodal curves where the solutions for each initial condition disappears in Figs. 1(a)-1(c). Two solutions coexist between the two spinodal curves. From these figures, we can establish the existence of the first-order phase transition in the intermediate pattern ratio and under the weak strength of the transverse field. The green dotted curve denotes the critical 


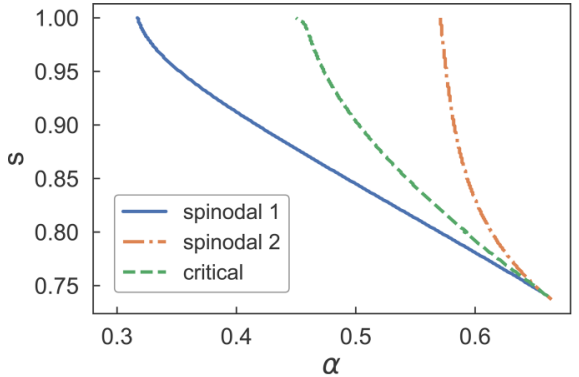

(a)

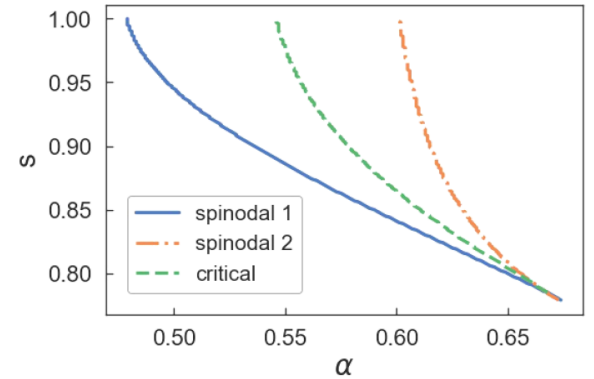

(b)

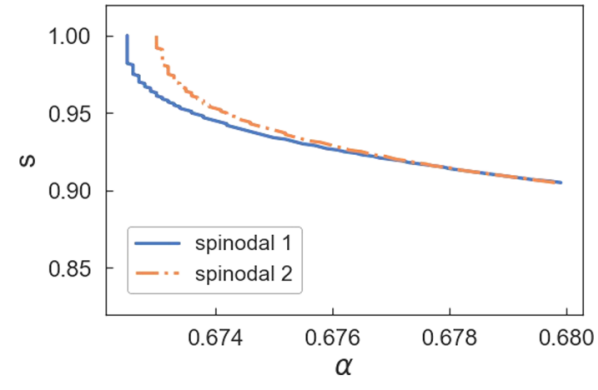

(c)

FIG. 1. Phase diagram of code-division multiple-access (CDMA) model in adiabatic reverse annealing (ARA) with $\lambda=1$. The horizontal axis denotes the pattern ratio. The vertical axis represents the annealing parameter. The experimental settings are (a) $T_{0}=0,(\mathrm{~b}) T_{0}=0.05$, and (c) $T_{0}=0.1$. The "spinodal 1" and "spinodal 2" curves indicate the solutions from the two different branches. The "critical" curve denotes the point at which the replica symmetry (RS) free energy takes the same value.

point at which the RS free energy takes the same value. In Fig. 1(c), we do not write down the curve because we cannot distinguish the critical point from the spinodal points in this scale. Higher noise results in a narrower region in which the two solutions coexist. Although the noise mitigates the first-order phase transition, it decreases the overlap between the original signal and the estimated one.

Next, we consider why the first-order phase transition is troublesome in estimating the original signal. The difficulty of estimating the original signal is related to the free energy landscape. We take Fig. 1(a) as an example. On the right side of spinodal curve 2 , it is easy to estimate the original signal because the free energy exhibits a minimum, which is a good estimator. When we set the pattern ratio as $\alpha=0.6$, we encounter the first-order phase transition at $s \simeq 0.8$. The free energy landscape has two valleys. At spinodal curve 2, the free energy landscape is transformed into a simple valley. In this case, it is comparatively easy to estimate the original signal. For $\alpha=0.5$, the spinodal curve 2 does not exist. The free energy landscape maintains two valleys. We cannot efficiently estimate the original signal because the metastable state remains. For $\alpha=0.4$, the critical point does not exist. In this case, we cannot obtain the original signal informationtheoretically. The ground state or low energy state does not

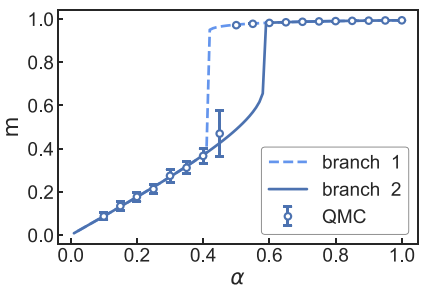

(a)

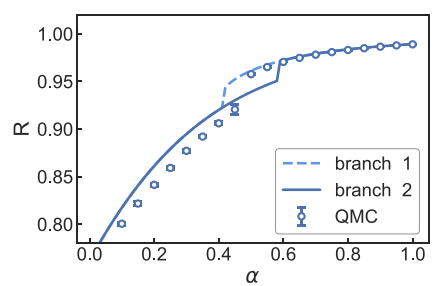

(b)
FIG. 2. Dependence of the order parameters on the pattern ratio for the fixed annealing parameter $s=0.9$. The vertical axes denote these order parameters: (a) magnetization and (b) correlation between Trotter slices. The solid blue and dashed blue curves denote the two different branches that are obtained from the saddle-point equations. The circles represent the results obtained by the quantum Monte Carlo simulations. correspond to the original signal at $s=1$. The minima of the free energy do not provide us with an effective estimation.

To verify the RS ansatz and the static approximation, we perform quantum Monte Carlo simulations for the CDMA model. We set the system size as $N=500$, the Trotter number as $M=50$, the temperature as $T=0.1$, and the true noise scale as $T_{0}=0$. We use a 100000 Monte Carlo step (MCS) average after $50000 \mathrm{MCS}$ equilibrations for each instance. We take the configuration average over the spreading codes and the original signals by randomly generating 50 instances. We plot the behavior of the order parameters with respect to the pattern ratio for the fixed annealing parameter $s=0.9$ in Fig. 2 and the annealing parameter for the fixed pattern ratio $\alpha=0.6$ in Fig. 3. The error bar is given by the standard deviation. The results obtained by the quantum Monte Carlo simulations are the averages over all Trotter slices. Following Ref. [66], we adopt the magnetization to quantify the performance of the MPM estimation. In this paper, we refer to the solution representing the "spinodal 1" curve as "branch 1" and to the solution representing the "spinodal 2" curve as "branch 2." According to Fig. 2, the results obtained by the quantum Monte Carlo simulations are consistent with the RS solutions, with the exception of the low pattern ratio. Figure 3 shows that the numerical results for the magnetization are consistent with the RS solutions, except for the intermediate values of the annealing parameter. The numerical result of the correlation between the Trotter slices does not follow the RS solutions other than the large annealing parameter, which is close to one [67]. To investigate the deviations between the numerical

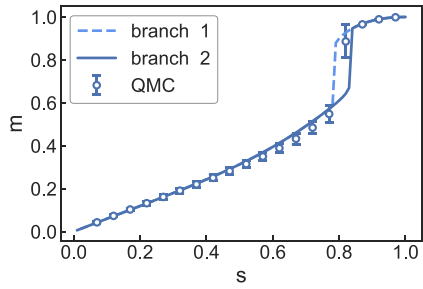

(a)

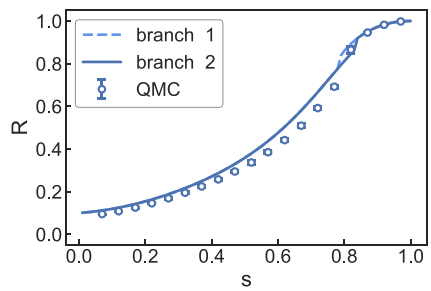

(b)
FIG. 3. Dependence of order parameters on annealing parameter for fixed pattern ratio $\alpha=0.6$. The same symbols as those in Fig. 2 are used. 


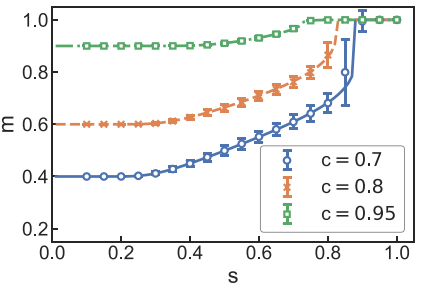

(a)

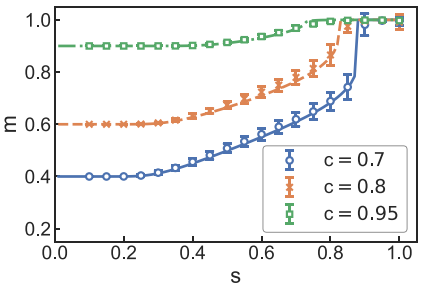

(b)

FIG. 4. Dependence of magnetization on annealing parameter with $\lambda=0$. The experimental settings are as follows: (a) $\alpha=0.6$ and $T_{0}=0$ and (b) $\alpha=0.62$ and $T_{0}=0.05$. Both axes are the same as those in Fig. 3(a).

results and the RS solutions due to replica symmetry breaking (RSB), we compute the Almeida-Thouless (AT) condition and the entropy. The details of these formulas are written in Appendix B. In these problem settings, the AT condition is not broken, and the entropy is positive. The deviations between the the numerical results and the RS solutions probably result from the breaking of the static approximation.

\section{ARA with $\lambda=0$}

To support the RS ansatz without the static approximation, we consider ARA with $\lambda=0$. In this case, the quantum part in Eq. (17) disappears. The experimental settings are the same as those in Fig. 3. We set $\alpha=0.6$ and $T_{0}=0$ in Fig. 4(a) and $\alpha=0.62$ and $T_{0}=0.05$ in Fig. 4(b). We consider three initial conditions: $c=0.7,0.8$, and 0.95 . The initial candidate solutions are generated from Eq. (19), given a fixed fraction $c$. The error bar is given by the standard deviation. Each curve represents the RS solutions, and each symbol denotes the numerical results obtained by the Markov-chain Monte Carlo simulations. It can be observed that the numerical results are consistent with the RS solutions. We can see that the deviations between the numerical results and the RS solutions are not the breaking of the RS ansatz to the breaking of the static approximation. For $\lambda=0$ with or without noise, ARA can avoid the first-order phase transition if we prepare for the proper initial conditions. In the next section, we analyze the general cases in detail.

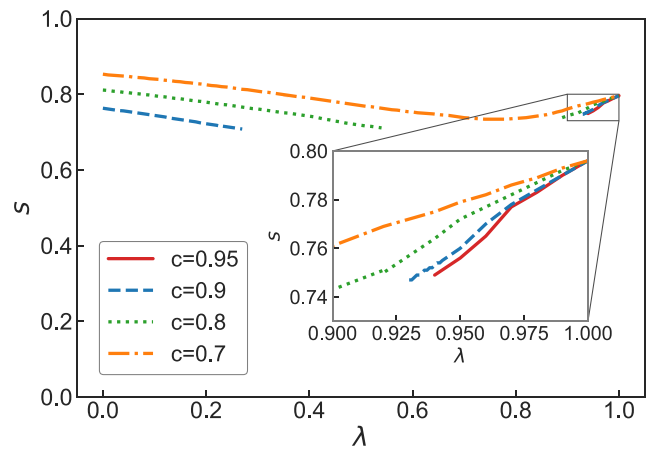

(a)

\section{ARA with finite $\lambda$}

We consider ARA with finite $\lambda$. The experimental settings are the same as those in Fig. 1(a). Figure 5 presents the phase diagram of the CDMA model in ARA for $\alpha=0.6$ and 0.5. We consider four initial conditions: $c=0.7,0.8,0.9$, and 0.95. Each curve represents a point of the first-order phase transition. We can observe from Figs. 5(a) and 5(b) that the first-order phase transition can be avoided if the initial state is close to the original signal. As the information regarding the original signal is increased, the region for avoiding the first-order phase transition is broadened. In Fig. 5(b), the region in which the first-order phase transition can be avoided is narrower than that in Fig. 5(a). For a lower pattern ratio, further information regarding the original signal is initially required to avoid the first-order phase transition. We also investigate the stability of the RS solutions and find that RSB does not happen for finite $\lambda$.

To analyze the extent to which the difficulty in obtaining the original signal is mitigated by ARA, we plot the differences in the magnetization $\Delta m$ between the two local minima at the first-order phase transition in the case of $\alpha=0.6$ and 0.5 in Fig. 6. As discussed in Ref. [57], the rate of quantum tunneling between two local minima in the free energy landscape is related to $\Delta m$. Figure 6 indicates that $\Delta m$ decreases as $c$ increases. For finite $\lambda, \Delta m$ is smaller than that of vanilla QA $(\lambda=1)$. Even though ARA cannot eliminate the first-order phase transition, the two local minima of the free energy become closer than those of the original one. The result demonstrates that ARA enhances the effects of quantum tunneling for the CDMA model. In ARA, we add the bias toward the original signal through the initial Hamiltonian. Since the bias removes or softens the free energy barrier, ARA can avoid or mitigate the first-order phase transition.

We consider the noise effects for the CDMA model in ARA. The experimental settings are the same as those illustrated in Fig. 1(b). Figure 7 displays the phase diagrams of CDMA in ARA for $\alpha=0.62$ and 0.57. The qualitative behaviors of the systems are approximately the same as those in the noiseless cases. The regions in which the first-order phase transition can be avoided are larger than those of the noiseless cases because the first-order phase transition is weakened

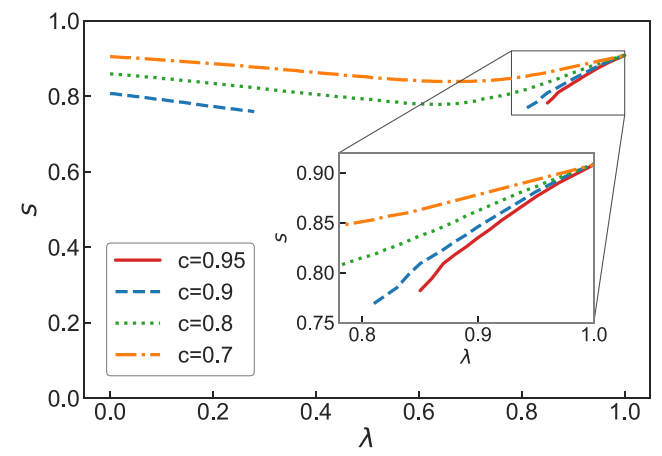

(b)

FIG. 5. Phase diagrams of the code-division multiple-access (CDMA) model in adiabatic reverse annealing (ARA) for four different values of $c$. The horizontal axis denotes the reverse annealing (RA) parameter. The vertical axis denotes the annealing parameter. These curves represent the points at which the first-order phase transitions occur. The experimental settings are (a) $\alpha=0.6$ and (b) $\alpha=0.5$. 


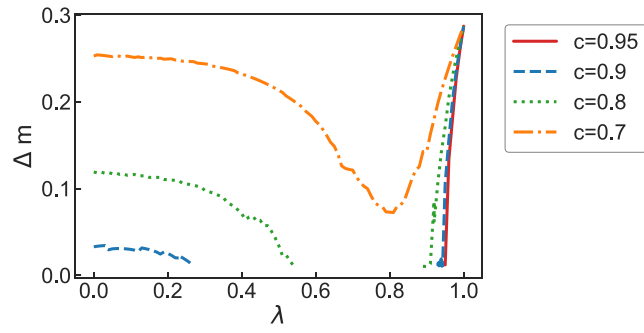

(a)

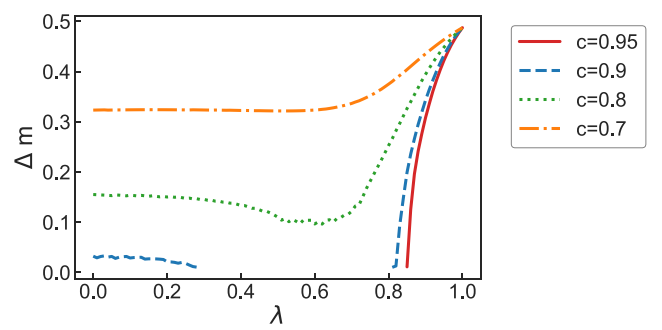

(b)

FIG. 6. Differences in magnetization between two local minima at first-order phase transition in Figs. 5(a) and 5(b). The vertical axis denotes the differences in the magnetization between the two local minima at the first-order phase transition. The horizontal axis denotes the reverse annealing (RA) parameter. The experimental settings are (a) $\alpha=0.6$ and (b) $\alpha=0.5$.

owing to the noise effects. Figure 8 presents $\Delta m$ in the case of $\alpha=0.62$ and 0.57 . We can see that $\Delta m$ is smaller than in the noiseless cases. In the small noisy cases, the free energy barrier is lower than in the noiseless cases. ARA works better in the small noisy cases than the noiseless cases.

To validate the RS solutions under the static approximation for finite $\lambda$, we perform quantum Monte Carlo simulations. The experimental settings are the same as those in Figs. 3 and 4. We set the RA parameter as $\lambda=0.8$, and the initial conditions as $c=0.7$ and 0.9. In Fig. 9, the order parameters for $c=0.7$ still exhibit a jump. In the case of $c=0.9$, it can be observed that the first-order phase transition can be avoided. We can see the deviations between the RS solutions, and the numerical results are the same as in Fig. 3. In these problem settings, RSB does not happen from the results of the saddle-point equations. Although the numerical results do not entirely match the RS solutions, the qualitative behaviors of the numerical results to avoid the first-order phase transition are like those of the RS solutions.

\section{B. Analysis of ARA in practical cases}

In Sec. IV A, we assume that the initial candidate solution is randomly generated from Eq. (19) with a fixed $c$. Practically, we need to prepare for the initial candidate solution by some algorithms. At first, we examine whether we can prepare for the proper initial condition to avoid the first-order phase transition with commonly used algorithms. Next, we evaluate the performance of ARA with the initial candidate solutions obtained by the algorithms.

\section{How to prepare for the initial candidate solution}

To prepare for the initial candidate solution, we adopt SA, simulated QA (SQA), and the approximate message passing (AMP) algorithm [68-70]. To perform SA and SQA, we take advantage of OpenJij, an open-source library for heuristic optimization problems in Python [71]. The implementation of the AMP algorithm is based on Ref. [69]. We perform three algorithms for 50 different instances. For SA and SQA, we carry out 51 different initial conditions for each instance. We set the system size as $N=8,16,32,64,128,256$, and 512 . We check the dependence of $c$ on $N$ with these algorithms in Fig. 10. We compute $c$ from the relationship $c=(1+\mathcal{M}) / 2$. We define the threshold required to avoid the first-order phase transition as $c_{\min }$. We calculate $c_{\min }$ from the saddle-point equations. We consider the region where the spinodal curve 2 does not exist and the critical curve exists, for example, $0.45 \leqslant \alpha \leqslant 0.57$ in Fig. 1 . In this region, to avoid or mitigate the first-order phase transition is crucial to estimate the original signal efficiently.

At first, we consider the noiseless case: $\alpha=0.5$ and $T_{0}=$ 0.0 . In this case, the threshold $c_{\text {min }} \approx 0.816$. Figure 10 (a) shows that the results of the three algorithms almost converge to the RS solutions $c_{\text {replica }} \simeq 0.864$ as we increase $N$. These practical algorithms can lead to the candidate solutions exceeding $c_{\min }$. Next, we consider the noisy case: $\alpha=0.57$ and

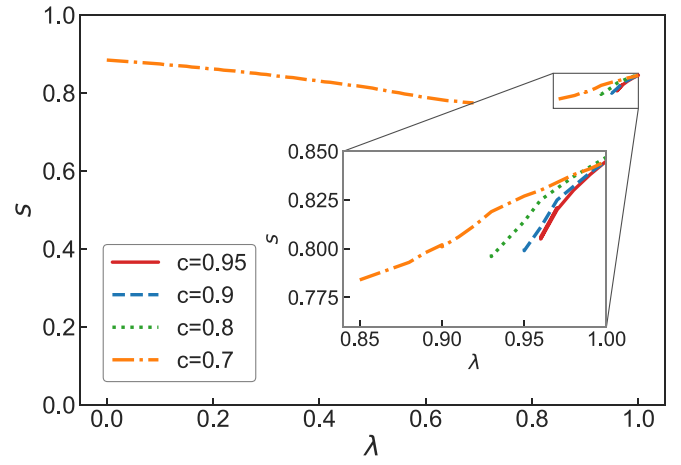

(a)

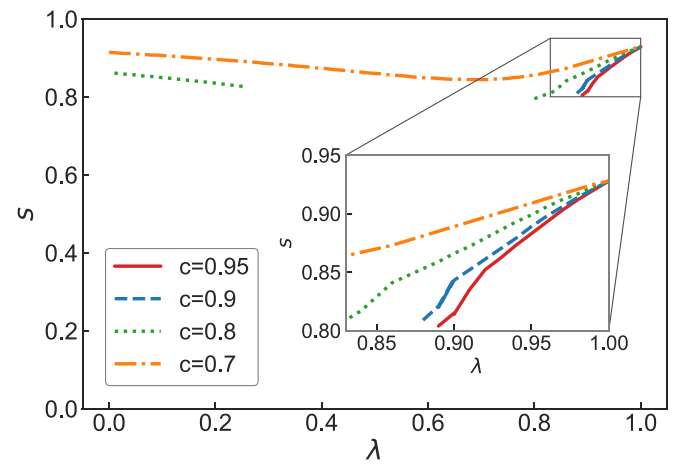

(b)

FIG. 7. Phase diagrams of the code-division multiple-access (CDMA) model in adiabatic reverse annealing (ARA) for four different values of $c$. Both axes are the same as those in Fig. 5. The experimental settings are (a) $\alpha=0.62$ and (b) $\alpha=0.57$. 


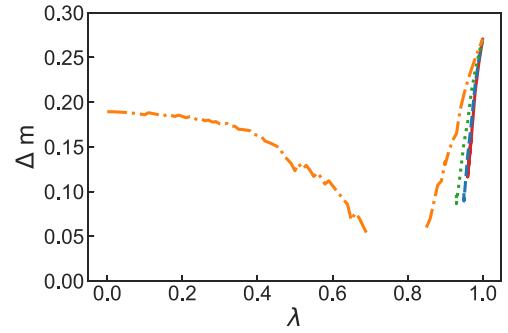

(a)
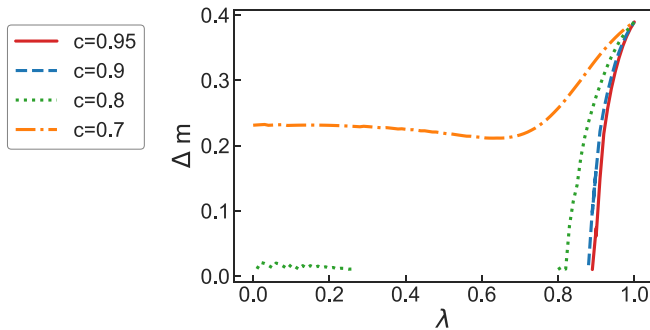

(b)

FIG. 8. The differences of the magnetization between the two local minima at the first-order phase transition in Figs. 6(a) and 6(b). Both axes are the same as those in Fig. 6. The experimental settings are (a) $\alpha=0.62$ and (b) $\alpha=0.57$.

$T_{0}=0.05$. The threshold $c_{\min } \approx 0.756$. Figure $10(\mathrm{~b})$ exhibits that these practical algorithms can accomplish $c_{\min }$ in the noisy case.

\section{Performance evaluation}

We evaluate the performance of ARA with the initial candidate solution attained by the practical algorithms. We adopt the AMP algorithm to prepare for the initial candidate solution. The experimental settings are the same as those in Fig. 3. We set the RA parameter as $\lambda=0.6$. At first, we perform the AMP algorithm for each instance. We utilize the final result as the initial candidate solution. We call this setting "AMP init." For the same instance, we randomly generate the initial candidate solution from Eq. (19), with a fixed fraction $c$, which is the same as one obtained by the AMP algorithm. We call this setting "random init."

We plot the magnetization for two initializations in Fig. 11. The error bar is given by the standard deviation. We consider two cases: $\alpha=0.5$ and $T_{0}=0$ in Fig. 11(a) and $\alpha=0.57$ and $T_{0}=0.05$ in Fig. 11(b). The dashed curves represent the RS solutions with $c_{\text {replica }}$. In the random init. setting, the numerical results are consistent with the RS solutions. In this setting, the first-order phase transition can be avoided by ARA with and without noise. In the AMP init. setting, the numerical results do not match the RS solutions. ARA cannot exclude the first-order phase transition. In Fig. 12, we plot the histogram of the magnetization at $s=0.99$ used in Fig. 11 to check the existence of the first-order phase transition in detail. In the random init. setting, only one peak exists at $m \simeq 1$. In the AMP init. setting, there are two peaks around $m \simeq 1$ and $m \neq 1$. ARA cannot eliminate the first-order phase transition even though the fraction $c$ obtained by the AMP algorithm exceeds the threshold $c_{\min }$. For the other practical algorithms, similar behaviors seem to occur.

The deviations between the numerical results of ARA in the AMP init. setting and the RS solutions are due to the assumption of the probability distribution of the initial candidate solution in our replica analysis. We assume that the probability distribution of the initial candidate solution follows Eq. (19). Since the initial candidate solution obtained by the AMP algorithm is not generated from Eq. (19), we cannot directly apply our analytical results to the AMP init. setting.

Finally, we consider why ARA in the random init. setting can avoid the first-order phase transition and ARA in the AMP init. setting cannot. In the AMP init. setting, the initial candidate solution depends on the original signal, the received signal, and the spreading codes. Therefore, the initial candidate solution is correlated with the received signal and the spreading codes. Meanwhile, in the random init. setting, the initial candidate solution depends only on the original signal and does not depend on the received signal and the spreading codes. The information about the original signal can be attained through the received signal and the spreading codes. In the random init. setting, the initial candidate solution does not have the information about the original signal included in the received signal and the spreading codes. The initial candidate solution increases the effective pattern ratio. As we increase the pattern ratio, the free energy barrier gets smaller. Thus, ARA in the random init. setting can avoid the

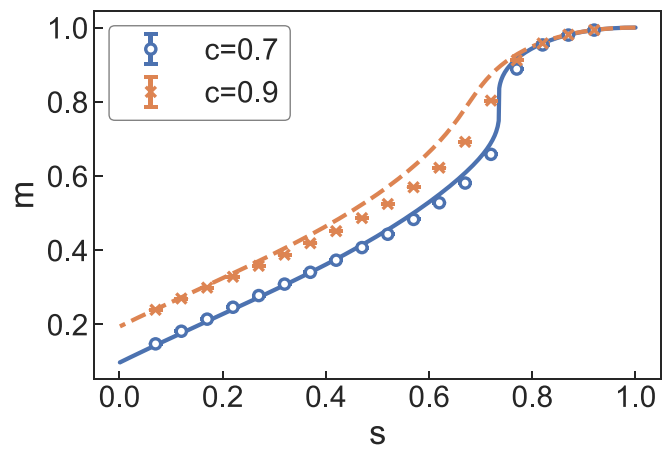

(a)

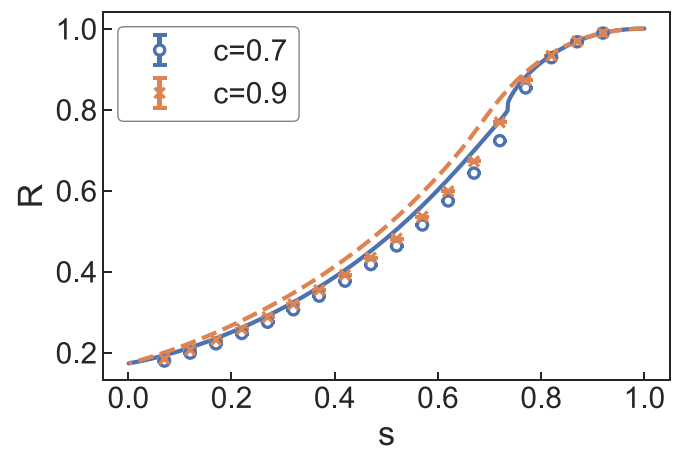

(b)

FIG. 9. Dependence of order parameters on annealing parameter with $\lambda=0.8$. Both axes are the same as those in Fig. 3 . 


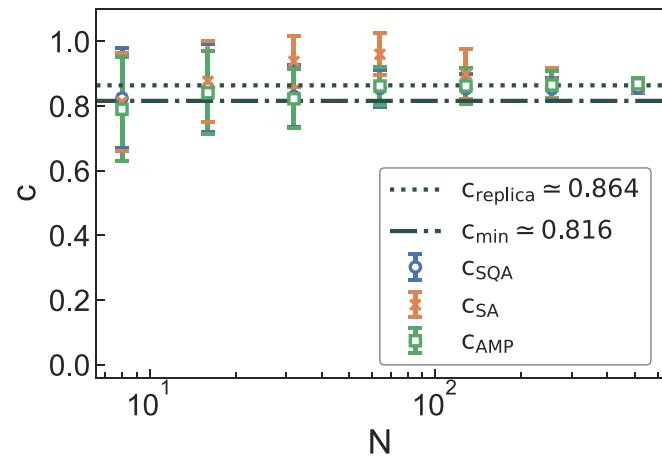

(a)

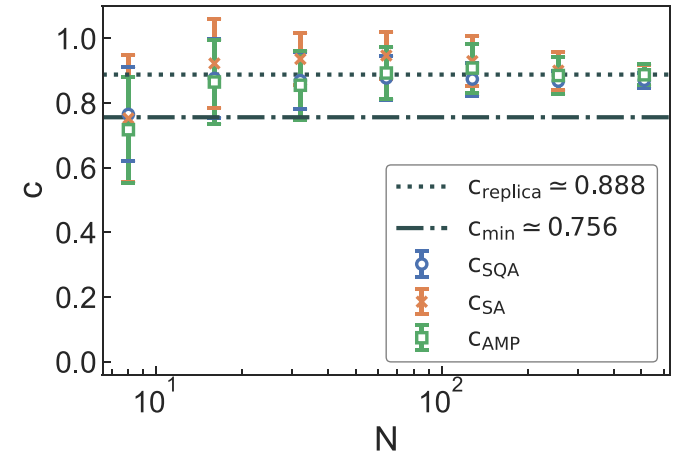

(b)

FIG. 10. The dependence of the fraction of the ground state in the estimated signal obtained by simulated annealing (SA), simulated quantum annealing (SQA), and the approximate message passing (AMP) algorithm on the system size. The experimental settings are as follows: (a) $\alpha=0.5$ and $T_{0}=0.0$ and (b) $\alpha=0.57$ and $T_{0}=0.05$.

first-order phase transition. In the AMP init. setting, the initial candidate solution only has the same information about the original signal obtained through the received signal and the spreading codes. Since the effective pattern ratio is the same as the original one, the free energy landscape in the AMP init. setting is the same as the original one. Consequently, ARA in the AMP init. setting cannot eliminate the first-order phase transition even if the candidate solution obtained by the AMP algorithm exceeds $c_{\text {min }}$, which is attained from the saddlepoint equations in oracle cases. To analyze the performance of ARA in the AMP init. setting appropriately, we should change the probability distribution of the initial candidate solution in our replica analysis.

\section{CONCLUSIONS}

We performed a mean-field analysis of ARA for CDMA multiuser detection. In CDMA multiuser detection, the firstorder phase transition is encountered in the intermediate pattern ratio. This first-order phase transition degrades the estimation performance. To avoid the first-order phase transition, we applied ARA to CDMA multiuser detection.

Firstly, we considered ARA in oracle cases, where the initial candidate solution is randomly generated from the original signal with a fixed fraction $c$. The first-order phase transition can be avoided by ARA if we prepare for the proper initial condition. In ARA, the differences in the magnetization between the two local minima at the first-order phase transition were smaller than those in vanilla QA. The prior information of the original signal avoids or mitigates the first-order phase transition. To validate our analysis, we performed quantum Monte Carlo simulations. The numerical results were consistent with the RS solutions under the static approximation, except for the intermediate values of the annealing parameter. Although the RS solutions under the static approximation were invalid in these cases, the results obtained from the RS solutions that ARA can avoid the first-order phase transition were consistent with the numerical results. The RS solutions under the static approximation can be useful for understanding the qualitative behaviors of ARA.

Next, we considered ARA in practical cases where we prepare for the initial candidate solution by the practical algorithms. We considered the three algorithms: SA, SQA, and the AMP algorithms. The fraction $c$ obtained by these practical algorithms can exceed the threshold $c_{\min }$ to avoid the firstorder phase transition. To evaluate the performance of ARA with the initial candidate solution attained by the practical algorithms, we performed quantum Monte Carlo simulations.

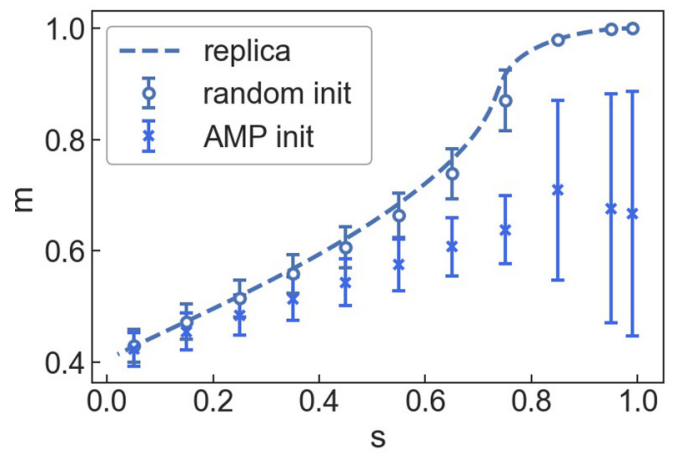

(a)

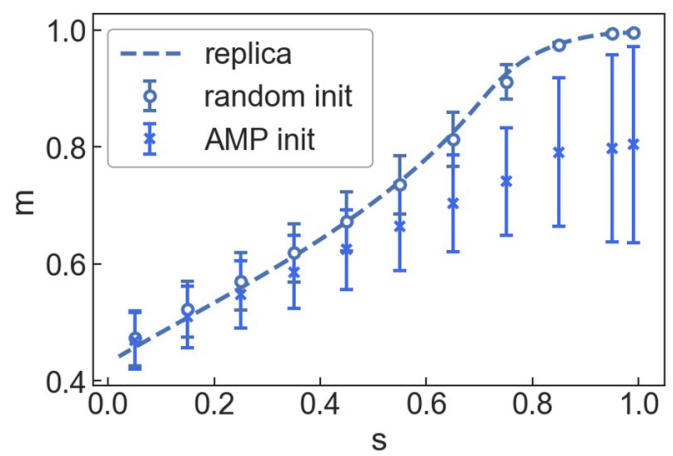

(b)

FIG. 11. Dependence of magnetization on annealing parameter with $\lambda=0.6$ in the "random init." and the "AMP init." settings. The dashed curve for each case is obtained by the saddle-point equations. The experimental settings are as follows: (a) $\alpha=0.5$ and $T_{0}=0$ and (b) $\alpha=0.57$ and $T_{0}=0.05$. Both axes are the same as those in Fig. 3(a). 


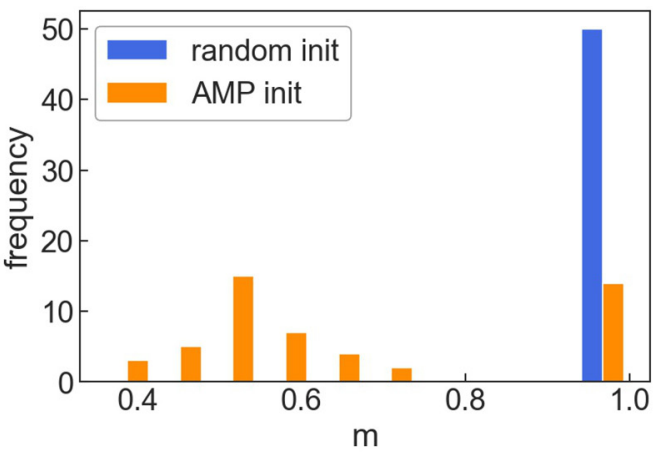

(a)

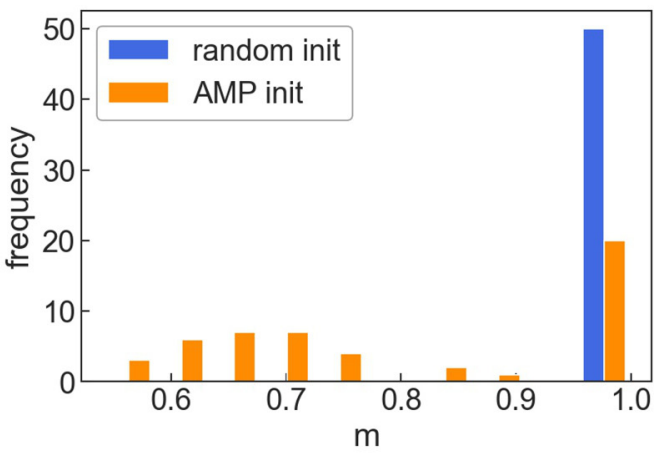

(b)

FIG. 12. Histogram of magnetization in the "random init." and the "AMP init." settings at $s=0.99$ for 50 instances in Fig. 11 . The experimental settings are as follows: (a) is $\alpha=0.5$ and $T_{0}=0$, and (b) is $\alpha=0.57$ and $T_{0}=0.05$.

In the AMP init. setting, we prepared for the initial candidate solution with the AMP algorithm. To compare with the AMP init. setting, we considered the random init. setting, where the initial candidate solution was randomly generated from Eq. (19), with a fixed fraction $c$, which was the same as the one obtained by the AMP algorithm for each instance. ARA in the random init. setting can utilize the additional information about the original signal not included in the received signal and the spreading codes. Since the free energy barrier is removed by the additional information about the original signal, ARA in the random init.setting can avoid the first-order phase transition. Meanwhile, in the AMP init. setting, the initial candidate solution was correlated with the received signal and the spreading codes. The initial candidate solution only had the same information attained through the received signal and the spreading codes. Because no additional information about the original signal existed, the effective free energy landscape was the same as the original one. Therefore, ARA in the AMP init. setting cannot avoid the first-order phase transition. ARA in the AMP init. setting did not match the RS solutions, whereas ARA in the random init. setting matched. The deviations between the numerical result of ARA in the AMP init. setting and the RS solutions were due to the assumption of the probability distribution of the initial candidate solution in our replica analysis.

In the AMP init. setting, the initial candidate solution was correlated with the received signal and the spreading codes. To incorporate the correlation with the received signal and the spreading codes into the initial candidate solution, we need to consider an equilibrium configuration governed by the GibbsBoltzmann distribution. Then the free energy is constrained by the equilibrium configuration. The equilibrium property of the constrained free energy can be analyzed by the Franz-Parisi potential, which is developed to study the metastable state structure for discontinuous mean-field spin glasses [72-76]. In a future study, we will analyze the Franz-Parisi potential for CDMA to investigate the performance of ARA in practical cases properly.

Although we cannot directly apply our theoretical results to the practical cases, we showed that ARA in the random init. setting can avoid the first-order phase transition for CDMA multiuser detection. We exhibited that the probability distribution of the initial candidate solution was crucial in ARA.
Our results indicated that the effective free energy landscape did not change by ARA with the initial candidate solution obtained by some practical algorithms. In practical cases, ARA did not enhance the estimation performance for CDMA multiuser detection. Similar behaviors would occur when we apply ARA to combinatorial optimization problems.

\section{ACKNOWLEDGMENTS}

M.O. was supported by KAKENHI (No. $19 \mathrm{H} 01095$ and No. 20H02168), the Next Generation High-Performance Computing Infrastructures and Applications R\&D Program by MEXT, and MEXT-Quantum Leap Flagship Program Grant No. JPMXS0120352009. K.T. was supported by JSPS KAKENHI (No. 18H03303). This paper was partly supported by JST-CREST (No. JPMJCR1402).

\section{APPENDIX A: DERIVATION OF FREE ENERGY}

We derive the free energy density under the RS ansatz and static approximation. Our derivation is based on a previous study [12]. We introduce the following terms:

$$
\begin{aligned}
u_{0}^{\mu} & =\frac{1}{\sqrt{N}} \sum_{i=1}^{N} \eta_{i}^{\mu} \xi_{i}, \\
u_{a}^{\mu}(t) & =\frac{1}{\sqrt{N}} \sum_{i=1}^{N} \eta_{i}^{\mu} \sigma_{i a}(t) .
\end{aligned}
$$

First, we insert the identity by using the $\delta$ function as

$$
\begin{gathered}
\int d u_{0}^{\mu} \delta\left(u_{0}^{\mu}-\frac{1}{\sqrt{N}} \sum_{i=1}^{N} \eta_{i}^{\mu} \xi_{i}\right)=1, \\
\int d u_{a}^{\mu}(t) \delta\left[u_{a}^{\mu}(t)-\frac{1}{\sqrt{N}} \sum_{i=1}^{N} \eta_{i}^{\mu} \sigma_{i a}(t)\right]=1,
\end{gathered}
$$

into the expression of the partition function after performing the ST decomposition. We here use the Fourier integral representation of the $\delta$ function and then introduce the integral variables as $\tilde{u}_{0}^{\mu}$ and $\tilde{u}_{a}^{\mu}(t)$. The replicated partition function 
can be thus rewritten as

$$
\begin{aligned}
{\left[Z^{n}\right]=} & \lim _{M \rightarrow \infty} \sum_{\left\{\eta_{i}^{\mu}= \pm 1\right\}} \sum_{\left\{\xi_{i}= \pm 1\right\}} \sum_{\left\{\tau_{i}= \pm \xi_{i}\right\}} P(\boldsymbol{\eta}) P(\boldsymbol{\xi}) P(\boldsymbol{\tau})\left(\sqrt{\frac{\beta_{0}}{2 \pi}}\right)^{K} \int d \mathbf{y} \prod_{\mu=1}^{K} \exp \left[-\frac{\beta_{0}}{2}\left(y^{\mu}-u_{0}^{\mu}\right)^{2}\right] \\
& \times\left(\prod_{\mu=1}^{K} \int \frac{d u_{0}^{\mu} d \tilde{u}_{0}^{\mu}}{2 \pi}\right) \prod_{\mu=1}^{K} \exp \left[i \tilde{u}_{0}^{\mu}\left(u_{0}^{\mu}-\frac{1}{\sqrt{N}} \sum_{i=1}^{N} \eta_{i}^{\mu} \xi_{i}\right)\right] \\
& \times\left[\prod_{\mu, a, t} \int \frac{d u_{a}^{\mu}(t) d \tilde{u}_{a}^{\mu}(t)}{2 \pi}\right]\left(\prod_{\mu, a, t} \exp \left\{i \tilde{u}_{a}^{\mu}(t)\left[u_{a}^{\mu}(t)-\frac{1}{\sqrt{N}} \sum_{i=1}^{N} \eta_{i}^{\mu} \sigma_{i a}(t)\right]\right\}\right) \\
& \times \exp \left\{-\frac{\beta s}{2 M} \sum_{\mu, a, t}\left[u_{a}^{\mu}(t)\right]^{2}+\frac{\beta s}{M} \sum_{\mu, a, t} y^{\mu} u_{a}^{\mu}(t)\right\} \\
& \times \operatorname{Tr}\left\{\frac{1}{2} \sinh \left[\frac{2 \beta(1-s) \lambda}{M}\right]\right\}^{\frac{n M N}{2}} \exp \left\{\frac{\beta(1-s)(1-\lambda)}{M} \sum_{a, t, i} \tau_{i} \sigma_{i a}(t)+\frac{1}{2} \ln \operatorname{coth}\left[\frac{\beta(1-s) \lambda}{M}\right] \sum_{a, t, i} \sigma_{i a}(t) \sigma_{i a}(t+1)\right\} .
\end{aligned}
$$

We perform the average over the spreading codes $\boldsymbol{\eta}$ in Eq. (A5) as follows:

$$
\begin{aligned}
\frac{1}{2^{N K}} \sum_{\left\{\eta_{i}^{\mu}= \pm 1\right\}} \prod_{\mu, i} \exp \left\{-\frac{i}{\sqrt{N}}\left[\tilde{u}_{0}^{\mu} \xi_{i}+\sum_{a, t} \tilde{u}_{a}^{\mu}(t) \sigma_{i a}(t)\right] \eta_{i}^{\mu}\right\} & =\frac{1}{2^{N K}} \prod_{\mu, i}\left(2 \cosh \left\{\frac{i}{\sqrt{N}}\left[\tilde{u}_{0}^{\mu} \xi_{i}+\sum_{a, t} \tilde{u}_{a}^{\mu}(t) \sigma_{i a}(t)\right]\right\}\right) \\
& =\prod_{\mu, i}\left(\cos \left\{\frac{1}{\sqrt{N}}\left[\tilde{u}_{0}^{\mu} \xi_{i}+\sum_{a, t} \tilde{u}_{a}^{\mu}(t) \sigma_{i a}(t)\right]\right\}\right) \\
& =\exp \left(\sum_{\mu, i} \ln \cos \left\{\frac{1}{\sqrt{N}}\left[\tilde{u}_{0}^{\mu} \xi_{i}+\sum_{a, t} \tilde{u}_{a}^{\mu}(t) \sigma_{i a}(t)\right]\right\}\right) \\
& \simeq \exp \left\{-\frac{1}{2 N} \sum_{\mu, i}\left[\tilde{u}_{0}^{\mu} \xi_{i}+\sum_{a, t} \tilde{u}_{a}^{\mu}(t) \sigma_{i a}(t)\right]^{2}\right\}
\end{aligned}
$$

where we utilize these relationships as $\cosh (i x)=\cos (x)$ and $\ln \cos (x) \simeq-x^{2} / 2$. Here, we find the order parameters in the expression of the replicated partition function. Again, we introduce the $\delta$ function and its Fourier integral representation for the order parameters as follows:

$$
\begin{gathered}
\prod_{a, t} \int d m_{a}(t) \delta\left[m_{a}(t)-\frac{1}{N} \sum_{i=1}^{N} \xi_{i} \sigma_{i a}(t)\right] \\
=\prod_{a, t} \int \frac{i N d m_{a}(t) d \tilde{m}_{a}(t)}{2 \pi M} \exp \left\{-\frac{\tilde{m}_{a}(t)}{M}\left[N m_{a}(t)-\sum_{i=1}^{N} \xi_{i} \sigma_{i a}(t)\right]\right\}, \\
\prod_{a, t, t^{\prime}} \int d R_{a}\left(t, t^{\prime}\right) \delta\left[R_{a}\left(t, t^{\prime}\right)-\frac{1}{N} \sum_{i=1}^{N} \sigma_{i a}(t) \sigma_{i a}\left(t^{\prime}\right)\right] \\
=\prod_{a, t, t^{\prime}} \int \frac{i N d R_{a}\left(t, t^{\prime}\right) d \tilde{R}_{a}\left(t, t^{\prime}\right)}{2 \pi M^{2}} \exp \left\{-\frac{\tilde{R}_{a}\left(t, t^{\prime}\right)}{M^{2}}\left[N R_{a}\left(t, t^{\prime}\right)-\sum_{i=1}^{N} \sigma_{i a}(t) \sigma_{i a}\left(t^{\prime}\right)\right]\right\}, \\
\prod_{a<b, t, t^{\prime}} \int_{d} d q_{a b}\left(t, t^{\prime}\right) \delta\left[q_{a b}\left(t, t^{\prime}\right)-\frac{1}{N} \sum_{i=1}^{N} \sigma_{i a}(t) \sigma_{i b}\left(t^{\prime}\right)\right] \\
=\prod_{a<b, t, t^{\prime}} \int \frac{i N d q_{a b}\left(t, t^{\prime}\right) d \tilde{q}_{a b}\left(t, t^{\prime}\right)}{2 \pi M^{2}} \exp \left\{-\frac{\tilde{q}_{a b}\left(t, t^{\prime}\right)}{M^{2}}\left[N q_{a b}\left(t, t^{\prime}\right)-\sum_{i=1}^{N} \sigma_{i a}(t) \sigma_{i b}\left(t^{\prime}\right)\right]\right\} .
\end{gathered}
$$


After inserting Eqs. (A7)-(A9) into Eq. (A5), the replicated partition function can be represented as

$$
\begin{aligned}
{\left[Z^{n}\right]=} & \lim _{M \rightarrow \infty}\left[\prod_{a, t} \int \frac{i N d m_{a}(t) d \tilde{m}_{a}(t)}{2 \pi M}\right]\left[\prod_{a, t, t^{\prime}} \int \frac{i N d R_{a}\left(t, t^{\prime}\right) d \tilde{R}_{a}\left(t, t^{\prime}\right)}{2 \pi M^{2}}\right]\left[\prod_{a<b, t, t^{\prime}} \int \frac{i N d q_{a b}\left(t, t^{\prime}\right) d \tilde{q}_{a b}\left(t, t^{\prime}\right)}{2 \pi M^{2}}\right] e^{G_{1}+G_{2}+G_{3},},(\mathrm{~A} 10) \\
e^{G_{1}} \equiv & \left(\prod_{\mu=1}^{K} \int \frac{d u_{0}^{\mu} d \tilde{u}_{0}^{\mu}}{2 \pi}\right)\left[\prod_{\mu, a, t} \int \frac{d u_{a}^{\mu}(t) d \tilde{u}_{a}^{\mu}(t)}{2 \pi}\right]\left[\prod_{\mu=1}^{K}\left(\frac{\beta_{0}}{2 \pi}\right)^{\frac{1}{2}} \int d y^{\mu}\right] \\
\times & \prod_{\mu=1}^{K} \exp \left(-\frac{\beta_{0}}{2}\left(y^{\mu}-u_{0}^{\mu}\right)^{2}+i \sum_{a, t} \tilde{u}_{a}^{\mu}(t) u_{a}^{\mu}(t)+i \tilde{u}_{0}^{\mu} u_{0}^{\mu}-\frac{1}{2}\left(\tilde{u}_{0}^{\mu}\right)^{2}-\tilde{u}_{0}^{\mu} \sum_{a, t} \tilde{u}_{a}^{\mu}(t) m_{a}(t)-\sum_{a<b} \sum_{t, t^{\prime}} \tilde{u}_{a}^{\mu}(t) \tilde{u}_{b}^{\mu}\left(t^{\prime}\right) q_{a b}\left(t, t^{\prime}\right)\right. \\
- & \left.\frac{1}{2} \sum_{a=1}^{n} \sum_{t, t^{\prime}} \tilde{u}_{a}^{\mu}(t) \tilde{u}_{a}^{\mu}\left(t^{\prime}\right) R_{a}\left(t, t^{\prime}\right)-\frac{\beta s}{2 M} \sum_{a, t}\left\{\left[u_{a}^{\mu}(t)\right]^{2}-2 y^{\mu} u_{a}^{\mu}(t)\right\}\right), \\
& e^{G_{2}} \equiv \sum_{\left\{\xi_{i}= \pm 1\right\}} \sum_{\left\{\tau_{i}= \pm \xi_{i}\right\}} P(\xi) P(\tau) \operatorname{Tr}\left\{\frac{1}{2} \sinh \left[\frac{2 \beta(1-s) \lambda}{M}\right]\right\}^{\frac{n M N}{2}} \\
& \times \exp \left\{\frac{1}{M} \sum_{a, t} \tilde{m}_{a}(t) \sum_{i=1}^{N} \xi_{i} \sigma_{i a}(t)+\frac{\beta(1-s)(1-\lambda)}{M} \sum_{a, t, i} \tau_{i} \sigma_{i a}(t)+\frac{1}{M^{2}} \sum_{a, t, t^{\prime}} \tilde{R}_{a}\left(t, t^{\prime}\right) \sum_{i=1}^{N} \sigma_{i a}(t) \sigma_{i a}\left(t^{\prime}\right)\right. \\
& \left.+\frac{1}{M^{2}} \sum_{a<b} \sum_{t, t^{\prime}} \tilde{q}_{a b}\left(t, t^{\prime}\right) \sum_{i=1}^{N} \sigma_{i a}(t) \sigma_{i b}\left(t^{\prime}\right)+\frac{1}{2} \ln \operatorname{coth}\left[\frac{\beta(1-s) \lambda}{M}\right] \sum_{a, t, i} \sigma_{i a}(t) \sigma_{i a}(t+1)\right\}, \\
& e^{G_{3}} \equiv \exp \left\{-\frac{N}{M}\left[\sum_{a, t} \tilde{m}_{a}(t) m_{a}(t)+\frac{1}{M} \sum_{a, t, t^{\prime}} \tilde{R}_{a}\left(t, t^{\prime}\right) R_{a}\left(t, t^{\prime}\right)+\frac{1}{M} \sum_{a<b} \sum_{t, t^{\prime}} \tilde{q}_{a b}\left(t, t^{\prime}\right) q_{a b}\left(t, t^{\prime}\right)\right]\right\} .
\end{aligned}
$$

We compute $e^{G_{1}}, e^{G_{2}}$, and $e^{G_{3}}$ individually. Firstly, we perform integration for $u_{0}^{\mu}$ and $\tilde{u}_{0}^{\mu}$ in Eq. (A11), and we can obtain $e^{G_{1}}$ as follows:

$$
\begin{aligned}
e^{G_{1}} & =\left[\prod_{\mu, a, t} \int \frac{d u_{a}^{\mu}(t) d \tilde{u}_{a}^{\mu}(t)}{2 \pi}\right]\left[\prod_{\mu=1}^{K}\left(\frac{1}{2 \pi} \sqrt{\frac{2 \beta_{0} \pi}{1+\beta_{0}}}\right) \int d y^{\mu}\right] \\
& \times \prod_{\mu=1}^{K} \exp \left\{\frac{\beta_{0}}{2\left(1+\beta_{0}\right)}\left[i y^{\mu}-\sum_{a, t} \tilde{u}_{a}^{\mu}(t) m_{a}(t)\right]^{2}+i \sum_{a, t} \tilde{u}_{a}^{\mu}(t) u_{a}^{\mu}(t)-\sum_{a<b} \sum_{t, t^{\prime}} \tilde{u}_{a}^{\mu}(t) \tilde{u}_{b}^{\mu}\left(t^{\prime}\right) q_{a b}\left(t, t^{\prime}\right)\right. \\
& \left.-\frac{1}{2} \sum_{a, t, t^{\prime}} \tilde{u}_{a}^{\mu}(t) \tilde{u}_{a}^{\mu}\left(t^{\prime}\right) R_{a}\left(t, t^{\prime}\right)-\frac{\beta s}{2 M} \sum_{a, t}\left[\left(u_{a}^{\mu}(t)\right)^{2}-2 y^{\mu} u_{a}^{\mu}(t)\right]\right\} .
\end{aligned}
$$

In the above equation, the integration over $y^{\mu}$ can be performed as follows:

$$
\begin{gathered}
\left(\prod_{\mu=1}^{K} \int d y^{\mu}\right) \prod_{\mu=1}^{K} \exp \left\{-\frac{\beta_{0}}{2\left(1+\beta_{0}\right)}\left(y^{\mu}\right)^{2}+\left[\frac{\beta s}{M} \sum_{a, t} u_{a}^{\mu}(t)-\frac{i \beta_{0}}{1+\beta_{0}} \sum_{a, t} \tilde{u}_{a}^{\mu}(t) m_{a}(t)\right] y^{\mu}\right\} \\
=\left[\prod_{\mu=1}^{K} \sqrt{\frac{2 \pi\left(1+\beta_{0}\right)}{\beta_{0}}}\right] \prod_{\mu=1}^{K} \exp \left\{\frac{1+\beta_{0}}{2 \beta_{0}}\left[\frac{\beta s}{M} \sum_{a, t} u_{a}^{\mu}(t)-\frac{i \beta_{0}}{1+\beta_{0}} \sum_{a, t} \tilde{u}_{a}^{\mu}(t) m_{a}(t)\right]^{2}\right\} .
\end{gathered}
$$

Next, we take the integral over $u_{a}^{\mu}(t)$ as

$$
\begin{aligned}
& {\left[\prod_{\mu, a, t} \int d u_{a}^{\mu}(t)\right] \prod_{\mu=1}^{K} \exp \left\{-\frac{\beta s}{2 M} \sum_{a, t}\left[u_{a}^{\mu}(t)\right]^{2}+i \sum_{a, t} \tilde{u}_{a}^{\mu}(t) u_{a}^{\mu}(t)+\frac{\beta^{2} s^{2}\left(1+\beta_{0}\right)}{2 \beta_{0} M^{2}}\left[\sum_{a, t} u_{a}^{\mu}(t)\right]^{2}\right.} \\
& \left.\quad-\frac{i \beta s}{M}\left[\sum_{a, t} u_{a}^{\mu}(t)\right]\left[\sum_{a^{\prime}, t^{\prime}} \tilde{u}_{a^{\prime}}^{\mu}\left(t^{\prime}\right) m_{a^{\prime}}\left(t^{\prime}\right)\right]\right\} \\
& =\left[\prod_{\mu, a, t} \int d u_{a}^{\mu}(t)\right] \prod_{\mu=1}^{K} \int D v_{1} \prod_{a, t} \exp \left\{-\frac{\beta s}{2 M}\left[u_{a}^{\mu}(t)\right]^{2}+i \tilde{u}_{a}^{\mu}(t) u_{a}^{\mu}(t)+\frac{\beta s v_{1}}{M} \sqrt{\frac{1+\beta_{0}}{\beta_{0}}} u_{a}^{\mu}(t)\right.
\end{aligned}
$$




$$
\begin{aligned}
& \left.-\frac{i \beta s}{M} u_{a}^{\mu}(t)\left[\sum_{a^{\prime}, t^{\prime}} \tilde{u}_{a^{\prime}}^{\mu}\left(t^{\prime}\right) m_{a^{\prime}}\left(t^{\prime}\right)\right]\right\} \\
= & \left(\prod_{\mu, a, t} \sqrt{\frac{2 \pi M}{\beta s}}\right) \prod_{\mu=1}^{K} \int D v_{1} \prod_{a, t} \exp \left(\frac{M}{2 \beta s}\left\{\frac{\beta s v_{1}}{M} \sqrt{\frac{1+\beta_{0}}{\beta_{0}}}+i \tilde{u}_{a}^{\mu}(t)-\frac{i \beta s}{M}\left[\sum_{a^{\prime}, t^{\prime}} \tilde{u}_{a^{\prime}}^{\mu}\left(t^{\prime}\right) m_{a^{\prime}}\left(t^{\prime}\right)\right]\right\}\right),
\end{aligned}
$$

where we used the Hubbard-Stratonovich transformation to reduce the quadratic term $\left[\sum_{a, t} u_{a}^{\mu}(t)\right]^{2}$.

To obtain the detailed formulation of the free energy density, we assume that the RS ansatz and static approximation

$$
\begin{array}{lllll}
m_{a}(t)=m, & q_{a b}\left(t, t^{\prime}\right)=q & (a \neq b), & R_{a}\left(t, t^{\prime}\right)=R & \left(t \neq t^{\prime}\right), \\
\tilde{m}_{a}(t)=\tilde{m}, & \tilde{q}_{a b}\left(t, t^{\prime}\right)=\tilde{q} & (a \neq b), & \tilde{R}_{a}\left(t, t^{\prime}\right)=\tilde{R} & \left(t \neq t^{\prime}\right) .
\end{array}
$$

Equation (A14) can be expressed as

$$
\begin{aligned}
e^{G_{1}}= & \prod_{\mu} \int D v_{1} \int D v_{2} \prod_{a} \int_{0} D v_{3} \\
& \times\left(\prod _ { t } \sqrt { \frac { 2 \pi M } { \beta s } } \int \frac { d \tilde { u } _ { a } ^ { \mu } ( t ) } { 2 \pi } \operatorname { e x p } \left\{\frac{\beta s}{2 M} \frac{1+\beta_{0}}{\beta_{0}} v_{1}^{2}-\frac{M-\beta s(R-1)}{2 \beta s}\left[\tilde{u}_{a}^{\mu}(t)\right]^{2}\right.\right. \\
& \left.\left.+\left[i v_{1} \sqrt{\frac{1+\beta_{0}}{\beta_{0}}}(1-n \beta s m)+v_{2} \sqrt{2 m-q-n \beta s m^{2}}+v_{3} \sqrt{q-R}\right] \tilde{u}_{a}^{\mu}(t)\right\}\right),
\end{aligned}
$$

where we use the following relationships:

$$
\begin{gathered}
\sum_{a} \sum_{t, t^{\prime}} \tilde{u}_{a}^{\mu}(t) \tilde{u}_{a}^{\mu}\left(t^{\prime}\right) R_{a}\left(t, t^{\prime}\right)=R \sum_{a}\left[\sum_{t} \tilde{u}_{a}^{\mu}(t)\right]^{2}-(R-1) \sum_{a, t}\left[\tilde{u}_{a}^{\mu}(t)\right]^{2}, \\
\sum_{a<b} \sum_{t, t^{\prime}} \tilde{u}_{a}^{\mu}(t) \tilde{u}_{b}^{\mu}\left(t^{\prime}\right) q_{a b}\left(t, t^{\prime}\right)=\frac{q}{2}\left\{\left[\sum_{a, t} \tilde{u}_{a}^{\mu}(t)\right]^{2}-\sum_{a}\left[\sum_{t} \tilde{u}_{a}^{\mu}(t)\right]^{2}\right\},
\end{gathered}
$$

as well as the Hubbard-Stratonovich transformation on $\left(\sum_{a, t}\right)^{2}$ and $\sum_{a}\left(\sum_{t}\right)^{2}$.

In Eq. (A18), we perform integration over $\tilde{u}_{a}^{\mu}(t)$ as follows:

$$
\begin{aligned}
e^{G_{1}}= & \prod_{\mu} \int D v_{1} \int D v_{2} \prod_{a} \int D v_{3} \exp \left(\frac{\beta s}{2} \frac{1+\beta_{0}}{\beta_{0}} v_{1}^{2}\right)\left[\prod_{t} \sqrt{\frac{2 \pi M}{\beta s}} \int \frac{d \tilde{u}_{a}^{\mu}(t)}{2 \pi}\right] \\
& \times\left[\prod _ { t } \operatorname { e x p } \left(-\frac{M-\beta s(R-1)}{2 \beta s}\left\{\tilde{u}_{a}^{\mu}(t)-\frac{\beta s}{M-\beta s(R-1)}\right.\right.\right. \\
& \left.\left.\left.\times\left[i v_{1} \sqrt{\frac{1+\beta_{0}}{\beta_{0}}}(1-n \beta s m)+v_{2} \sqrt{2 m-q-n \beta s m^{2}}+v_{3} \sqrt{q-R}\right]\right\}\right)\right] \\
& \times \exp \left\{\frac{\beta s M}{2[M-\beta s(R-1)]}\left[i v_{1} \sqrt{\frac{1+\beta_{0}}{\beta_{0}}}(1-n \beta s m)+v_{2} \sqrt{2 m-q-n \beta s m^{2}}+v_{3} \sqrt{q-R}\right]^{2}\right\} \\
= & \prod_{\mu} \int D v_{1} \int D v_{2} \prod \int D v_{3} \exp \left(\frac{\beta s}{2} \frac{1+\beta_{0}}{\beta_{0}} v_{1}^{2}\right)\left[\prod \sqrt{\frac{M}{M-\beta s(R-1)}}\right] \\
& \times \exp \left\{\frac{\beta s M}{2[M-\beta s(R-1)]}\left[i v_{1} \sqrt{\frac{1+\beta_{0}}{\beta_{0}}}(1-n \beta s m)+v_{2} \sqrt{2 m-q-n \beta s m^{2}}+v_{3} \sqrt{q-R}\right]^{2}\right\} .
\end{aligned}
$$

In the limit of $M \rightarrow \infty$, we note that the coefficient terms in Eq. (A21) are reduced to

$$
\lim _{M \rightarrow \infty} \prod_{t} \sqrt{\frac{M}{M-\beta s(R-1)}}=\lim _{M \rightarrow \infty} \exp \left\{-\frac{M}{2} \ln \left[1-\frac{\beta s}{M}(R-1]\right)\right\} \simeq \exp \left[\frac{\beta s}{2}(R-1)\right],
$$




$$
\lim _{M \rightarrow \infty} \frac{\beta s M}{2[M-\beta s(R-1)]}=\frac{\beta s}{2},
$$

where we utilize the relationship as $\ln (1+x) \simeq x$.

We carry out integration over the Gaussian variables $v_{1}, v_{2}$, and $v_{3}$ as follows:

$$
\begin{aligned}
e^{G_{1}}= & \prod_{\mu} \int D v_{1} \int D v_{2} \exp \left[\frac{\beta n s}{2}(R-1)+\frac{\beta n s}{2} \frac{1+\beta_{0}}{\beta_{0}} v_{1}^{2}\right] \\
& \times \prod_{a} \int D v_{3} \exp \left\{\frac{\beta s}{2}\left[v_{3} \sqrt{q-R}+i v_{1} \sqrt{\frac{1+\beta_{0}}{\beta_{0}}}(1-n \beta s m)+v_{2} \sqrt{2 m-q-n \beta s m^{2}}\right]^{2}\right\} \\
= & \prod_{\mu} \int D v_{1} \int D v_{2}\left[\frac{1}{1+\beta s(R-q)}\right]^{\frac{n}{2}} \exp \left[\frac{\beta n s}{2}(R-1)\right] \\
& \times \exp \left(\frac{\beta n s}{2}\left\{\frac{1+\beta_{0}}{\beta_{0}} v_{1}^{2}+\frac{1}{1+\beta s(R-q)}\left[i v_{1} \sqrt{\frac{1+\beta_{0}}{\beta_{0}}}(1-n \beta s m)+v_{2} \sqrt{2 m-q-n \beta s m^{2}}\right]^{2}\right\}\right) \\
\simeq & \prod_{\mu}\left[\frac{1}{1+\beta s(R-q)}\right]^{\frac{n}{2}} \exp \left[\frac{\beta n s}{2}(R-1)\right] \\
& \times\left(1+\frac{\beta n s}{2} \int D v_{1} \int D v_{2}\left\{\frac{1+\beta_{0}}{\beta_{0}} v_{1}^{2}+\frac{1}{1+\beta s(R-q)}\left[i v_{1} \sqrt{\left.\left.\left.\frac{1+\beta_{0}}{\beta_{0}}(1-n \beta s m)+v_{2} \sqrt{2 m-q-n \beta s m^{2}}\right]^{2}\right\}\right)}\right.\right.\right. \\
= & \prod_{\mu}\left[\frac{1}{1+\beta s(R-q)}\right]^{\frac{n}{2}} \exp \left[\frac{\beta n s}{2}(R-1)\right]\left\{1+\frac{\beta n s}{2}\left[\frac{1+\beta_{0}}{\beta_{0}}+\frac{2 m-q-\left(1+\beta_{0}^{-1}\right)}{1+\beta s(R-q)}\right]+O\left(n^{2}\right)\right\} \\
\simeq & \exp \left(\frac{\alpha n N}{2}\left\{-\ln [1+\beta s(R-q)]+\beta s\left[(R-1)+\frac{1+\beta_{0}}{\beta_{0}}+\frac{2 m-q-\left(1+\beta_{0}^{-1}\right)}{1+\beta s(R-q)}\right]\right\}\right),
\end{aligned}
$$

where we use the Taylor expansion as $\exp (a x) \simeq 1+a x$ and omit the $O\left(n^{2}\right)$ term in Eq. (A24).

We calculate $e^{G_{2}}$ under the RS ansatz and static approximation as follows:

$$
\begin{aligned}
e^{G_{2}}= & \sum_{\left\{\xi_{i}= \pm 1\right\}} \sum_{\left\{\tau_{i}= \pm \xi_{i}\right\}} P(\xi) P(\boldsymbol{\tau}) \operatorname{Tr}\left\{\frac{1}{2} \sinh \left[\frac{2 \beta(1-s) \lambda}{M}\right]\right\}^{\frac{n M N}{2}} \int D z \\
& \times \exp \left\{\frac{\tilde{m}}{M} \sum_{a, t, i} \xi_{i} \sigma_{i a}(t)+\frac{\beta(1-s)(1-\lambda)}{M} \sum_{a, t, i} \tau_{i} \sigma_{i a}(t)+\frac{\sqrt{\tilde{q}}}{M} z \sum_{a, t, i} \sigma_{i a}(t)+\frac{2 \tilde{R}-\tilde{q}}{2 M^{2}} \sum_{a, i}\left[\sum_{t=1}^{M} \sigma_{i a}(t)\right]^{2}\right. \\
& \left.+\frac{1}{2} \ln \operatorname{coth}\left[\frac{\beta(1-s) \lambda}{M}\right] \sum_{a, t, i} \sigma_{i a}(t) \sigma_{i a}(t+1)\right\}, \\
= & \prod_{i=1}^{N} \sum_{\xi_{i}= \pm 1} \sum_{\tau_{i}= \pm \xi_{i}} \frac{1}{2} P\left(\tau_{i}\right) \int D z \prod_{a=1}^{n} \int D y \prod_{t=1}^{M} \operatorname{Tr}\left\{\frac{1}{2} \sinh \left[\frac{2 \beta(1-s) \lambda}{M}\right]\right\}^{\frac{1}{2}} \\
& \times \exp \left\{\frac{1}{M}\left[\tilde{m} \xi_{i}+\beta(1-s)(1-\lambda) \tau_{i}+\sqrt{2 \tilde{R}-\tilde{q} y}+\sqrt{\tilde{q} z}\right] \sigma_{i a}(t)+\frac{1}{2} \ln \operatorname{coth}\left[\frac{\beta(1-s) \lambda}{M}\right] \sigma_{i a}(t) \sigma_{i a}(t+1)\right\} \\
= & \prod_{i=1}^{N} \sum_{\xi_{i}= \pm 1} \sum_{\tau_{i}= \pm \xi_{i}} \frac{1}{2} P\left(\tau_{i}\right) \int D z\left\{\int D y 2 \cosh \sqrt{g^{2}\left(\tau_{i}, \xi_{i}\right)+[\beta(1-s) \lambda]^{2}}\right\}^{n} \\
\simeq & \prod_{i=1}^{N} \sum_{\xi_{i}= \pm 1} \frac{1}{2} \exp \left\{n \int D z \sum_{\tau_{i}= \pm \xi_{i}} P\left(\tau_{i}\right) \ln \int D y 2 \cosh \sqrt{g^{2}\left(\tau_{i}, \xi_{i}\right)+[\beta(1-s) \lambda]^{2}}\right\}
\end{aligned}
$$




$$
\begin{aligned}
& =\prod_{i=1}^{N} \frac{1}{2} \sum_{\xi_{i}= \pm 1} \exp \left(n\left\{\sum_{a= \pm 1} c_{a} \int D z \ln \int D y 2 \cosh \sqrt{g_{a}^{2}\left(\xi_{i}\right)+[\beta(1-s) \lambda]^{2}}\right\}\right) \\
& =\exp \left(n N\left\{\sum_{a= \pm 1} c_{a} \int D z \ln \int D y 2 \cosh \sqrt{g_{a}^{2}+[\beta(1-s) \lambda]^{2}}\right\}\right),
\end{aligned}
$$

where

$$
\begin{aligned}
& g\left(\tau_{i}, \xi_{i}\right)=\tilde{m} \xi_{i}+\beta(1-s)(1-\lambda) \tau_{i}+\sqrt{\tilde{q}} z+\sqrt{2 \tilde{R}-\tilde{q} y}, \\
& g_{a}\left(\xi_{i}\right)=[\tilde{m}+a \beta(1-s)(1-\lambda)] \xi_{i}+\sqrt{\tilde{q}} z+\sqrt{2 \tilde{R}-\tilde{q} y},
\end{aligned}
$$

in which we utilize the inverse operation of the ST decomposition and take the trace. Here, we use the notation $g_{a}\left(\xi_{i}=1\right)=g_{a}$.

Under the RS ansatz and static approximation, $e^{G_{3}}$ is expressed as

$$
e^{G_{3}}=\exp \left\{n N\left[-m \tilde{m}-m^{x} \tilde{m}^{x}-R \tilde{R}-\frac{n-1}{2} q \tilde{q}+O\left(\frac{1}{M}\right)\right]\right\} .
$$

In the limit of $M \rightarrow \infty$, the $O(1 / M)$ term is negligible.

The saddle-point method can be used in the thermodynamics limit $N \rightarrow \infty$, and the RS free energy density is expressed as

$$
\begin{aligned}
-\beta f_{\mathrm{RS}}= & \lim _{\substack{n \rightarrow 0 \\
N \rightarrow \infty}} \lim _{\left.N \rightarrow Z^{n}\right]-1} \\
= & \operatorname{extr}_{\substack{m, q, R \\
\tilde{m}, \tilde{q}, \tilde{R}}}\left(\frac{\alpha}{2}\left\{-\ln [1+\beta s(R-q)]+\beta s\left[(R-1)+\frac{1+\beta_{0}}{\beta_{0}}+\frac{2 m-q-\left(1+\beta_{0}^{-1}\right)}{1+\beta s(R-q)}\right]\right\}\right. \\
& \left.-m \tilde{m}-R \tilde{R}+\frac{1}{2} q \tilde{q}+\sum_{a= \pm 1} c_{a} \int D z \ln \int D y 2 \cosh \sqrt{g_{a}^{2}+[\beta(1-s) \lambda]^{2}}\right),
\end{aligned}
$$

where the order parameters and their conjugate parameters are decided by the saddle-point conditions in the free energy density. We neglect trivial terms and coefficients in Eq. (A29).

\section{APPENDIX B: THE SADDLE-POINT EQUATIONS AND THE STABILITY CONDITION OF THE RS SOLUTIONS}

We present the saddle-point equations and the stability condition of the RS solutions. The extremization of Eq. (A29) yields the following saddle-point equations:

$$
\begin{gathered}
m=\sum_{a= \pm 1} c_{a} \int D z Y_{a}^{-1} \int D y\left(\frac{g_{a}}{u_{a}}\right) \sinh u_{a} \\
q=\sum_{a= \pm 1} c_{a} \int D z\left[Y_{a}^{-1} \int D y\left(\frac{g_{a}}{u_{a}}\right) \sinh u_{a}\right]^{2} \\
R=\sum_{a= \pm 1} c_{a} \int D z Y_{a}^{-1} \int D y\left(\left\{\frac{[\beta(1-s) \lambda]^{2}}{u_{a}^{3}}\right\} \sinh u_{a}+\left(\frac{g_{a}}{u_{a}}\right)^{2} \cosh u_{a}\right), \\
\tilde{m}=\frac{\alpha \beta s}{1+\beta s(R-q)}, \\
\tilde{q}=\frac{\alpha \beta^{2} s^{2}\left(q-2 m+1+\beta_{0}^{-1}\right)}{[1+\beta s(R-q)]^{2}} \\
2 \tilde{R}-\tilde{q}=\frac{\alpha \beta^{2} s^{2}(R-q)}{1+\beta s(R-q)}, \\
Y_{a} \equiv \int D y \cosh u_{a}, \\
u_{a} \equiv \sqrt{g_{a}^{2}+[\beta(1-s) \lambda]^{2}} .
\end{gathered}
$$

The overlap can be written as

$$
\mathcal{M}=\sum_{a= \pm 1} c_{a} \int D z \operatorname{sgn}\left[Y_{a}^{-1} \int D y\left(\frac{g_{a}}{u_{a}}\right) \sinh u_{a}\right]
$$


The fraction of the ground state in the estimated signal is calculated by $c_{\text {repica }}=(1+\mathcal{M}) / 2$. Basically, we numerically assess the fixed points of the saddle-point equations by iterating the substitution of the tentative solutions. The fixed points are extrema of Eq. (A29) and characterize the free energy of the system.

Next, we consider the stability of the RS solutions. In the low-temperature regions, the classical CDMA model exhibits RSB [5]. Two instabilities exist in the RS solutions: the local and global instabilities of the RS solutions. The local stability condition of the RS solutions under the static approximation is expressed as

$$
\begin{aligned}
& \left.\left.\frac{\alpha \beta^{2} s^{2}}{[1+\beta s(R-q)]^{2}}\left\{\sum_{a= \pm 1} c_{a} \int D z \llbracket Y_{a}^{-1} \int D y\left(\frac{g_{a}}{u_{a}}\right) \sinh u_{a}\right]^{2}-Y_{a}^{-1}\left(\int D y\left\{\frac{[\beta(1-s) \lambda]^{2}}{u_{a}^{3}}\right\} \sinh u_{a}+\int D y\left(\frac{g_{a}}{u_{a}}\right)^{2} \cosh u_{a}\right)\right]^{2}\right\} \\
& <1 .
\end{aligned}
$$

This condition corresponds to the AT condition [77] in ARA. This result is consistent with the previous result in Ref. [5] for the classical limit $s=1$ and $\lambda=1$. We can attain this condition by considering the perturbations to the RS solutions [78]. The detailed calculations for deriving the AT condition in Eq. (B10) are presented in Appendix C. The global instability condition of the RS solutions is related to the negative entropy. The existence of the global instability corresponds to the freezing behavior [79]. To detect the freezing behavior, we calculate the RS entropy as follows:

$$
\begin{aligned}
\mathcal{S}=-\frac{\partial}{\partial T} f_{\mathrm{RS}}= & -\frac{\alpha}{2}\{\ln [1+\beta s(R-q)]\}+\frac{R-q}{2}(\tilde{m}-\tilde{q})+\tilde{R} R-\frac{1}{2} q \tilde{q} \\
& +\sum_{a= \pm 1} c_{a} \int D z \ln 2 Y_{a}-\beta\left(\sum_{a= \pm 1} c_{a} \int D z Y_{a}^{-1} \int D y u_{a} \sinh u_{a}\right) .
\end{aligned}
$$

In the case of $s=1$ and $\lambda=1$, this result is also consistent with the classical one.

\section{APPENDIX C: DERIVATION OF AT CONDITION}

We derive the AT condition for the CDMA model in ARA. The local stability of the RS solutions against the RSB perturbation is computed from the 1-step RSB (1RSB) solutions. The detailed derivation of the 1RSB solutions is as follows. Following Ref. [80], we assume the RS ansatz and static approximation for $m_{a}(t), R_{a}\left(t, t^{\prime}\right), \tilde{m}_{a}(t)$, and $\tilde{R}_{a}\left(t, t^{\prime}\right)$. For the spin glass order parameter and its conjugate parameter, we divide the replicas into two blocks and introduce two order parameters, as follows:

$$
q_{a_{l} b_{l}}\left(t, t^{\prime}\right)=\left\{\begin{array}{ll}
q_{0} & (l \notin \text { block }) \\
q_{1} & (l \in \text { block })
\end{array}, \quad \tilde{q}_{a_{l} b_{l}}\left(t, t^{\prime}\right)=\left\{\begin{array}{ll}
\tilde{q}_{0} & (l \notin \text { block }) \\
\tilde{q}_{1} & (l \in \text { block })
\end{array},\right.\right.
$$

where $l=1,2, \ldots, n / m_{1}$ is the block number, $m_{1}$ is Parisi's breaking parameter, and $a_{l}, b_{l}=1,2, \ldots, m_{1}$ is the index inside a block.

By using the 1RSB scheme, we can divide these terms in Eq. (A20) as follows:

$$
\sum_{a<b} \sum_{t, t^{\prime}} q_{a b}\left(t, t^{\prime}\right) \tilde{u}_{a}^{\mu}(t) \tilde{u}_{b}^{\mu}\left(t^{\prime}\right)=\frac{q_{0}}{2}\left[\sum_{l, a_{l}, t} \tilde{u}_{a_{l}}^{\mu}(t)\right]^{2}+\frac{q_{1}-q_{0}}{2} \sum_{l}\left[\sum_{a_{l}, t} \tilde{u}_{a_{l}}^{\mu}(t)\right]^{2}-\frac{q_{1}}{2} \sum_{l, a_{l}}\left[\sum_{t} \tilde{u}_{a_{l}}^{\mu}(t)\right]^{2} .
$$

Using Eq. (C2), we can rewrite $e^{G_{1}}$ as

$$
\begin{aligned}
e^{G_{1}}= & \prod_{\mu} \int D v_{1} \prod_{l, a_{l}, t} \frac{d \tilde{u}_{a_{l}}^{\mu}(t)}{2 \pi} \sqrt{\frac{2 \pi M}{\beta s}} \exp \left\{\frac{\beta s}{2 M} \frac{1+\beta_{0}}{\beta_{0}} v_{1}^{2}-\frac{M}{2 \beta s}\left[\tilde{u}_{a_{l}}^{\mu}(t)\right]^{2}-\frac{\beta s m^{2}}{2 M}\left[\sum_{l, a_{l}, t} \tilde{u}_{t}^{\mu}\left(a_{l}\right)\right]^{2}+i v_{1} \sqrt{\frac{1+\beta_{0}}{\beta_{0}}} \tilde{u}_{a_{l}}^{\mu}(t)\right. \\
& \left.+m \tilde{u}_{a_{l}}^{\mu}(t)\left[\sum_{l^{\prime}, a_{l}^{\prime}, t^{\prime}} \tilde{u}_{a_{l}^{\prime}}^{\mu}\left(t^{\prime}\right)\right]-i v_{1} \frac{\beta s m}{M} \sqrt{\frac{1+\beta_{0}}{\beta_{0}}}\left[\sum_{l^{\prime}, a_{l}^{\prime}, t^{\prime}} \tilde{u}_{a_{l}^{\prime}}^{\mu}\left(t^{\prime}\right)\right]\right\} \\
& \times \exp \left\{-\frac{q_{0}}{2}\left[\sum_{l, a_{l}, t} \tilde{u}_{a_{l}}^{\mu}(t)\right]^{2}-\frac{q_{1}-q_{0}}{2} \sum_{l}\left[\sum_{a_{l}, t} \tilde{u}_{a_{l}}^{\mu}(t)\right]^{2}+\frac{q_{1}}{2} \sum_{l, a_{l}}\left[\sum_{t} \tilde{u}_{a_{l}}^{\mu}(t)\right]^{2}-R \sum_{l, a_{l}}\left[\sum_{t} \tilde{u}_{a_{l}}^{\mu}(t)\right]^{2}(R-1) \sum_{l, a_{l}, t}\right. \\
& \left.\times\left[\tilde{u}_{a_{l}}^{\mu}(t)\right]^{2}\right\} \\
= & \prod_{\mu} \int D v_{1} \int D v_{2} \prod_{l} \int D v_{3} \prod_{a_{l}} \int D v_{4} \exp \left(\frac{\beta s v_{1}^{2}}{2} \frac{1+\beta_{0}}{\beta_{0}}\right) \prod_{t} \frac{d \tilde{u}_{a_{l}}^{\mu}(t)}{2 \pi} \sqrt{\frac{2 \pi M}{\beta s}} \\
& \times \exp \left\{-\frac{M-\beta s(R-1)}{2 \beta s}\left[\tilde{u}_{a_{l}}^{\mu}(t)\right]^{2}\right.
\end{aligned}
$$




$$
\begin{aligned}
& \left.+\left[i v_{1}(1-n \beta s m) \sqrt{\frac{1+\beta_{0}}{\beta_{0}}}+v_{2} \sqrt{2 m-q_{0}-n \beta s m^{2}}+v_{3} \sqrt{q_{0}-q_{1}}+v_{4} \sqrt{q_{1}-R}\right] \tilde{u}_{a_{l}}^{\mu}(t)\right\} \\
= & \prod_{\mu} \int D v_{1} \int D v_{2} \prod \int D v_{3} \prod \int D v_{a_{l}} \exp \left(\frac{\beta s v_{1}^{2}}{2} \frac{1+\beta_{0}}{\beta_{0}}\right)\left[\prod_{t} \sqrt{\frac{M}{M-\beta s(R-1)}}\right] \\
& \times \exp \left\{\frac{\beta s M}{2[M-\beta s(R-1)]}\left[i v_{1}(1-n \beta s m) \sqrt{\frac{1+\beta_{0}}{\beta_{0}}}+v_{2} \sqrt{2 m-q_{0}-n \beta s m^{2}}+v_{3} \sqrt{q_{0}-q_{1}}+v_{4} \sqrt{q_{1}-R}\right]^{2}\right\}
\end{aligned}
$$

where the Hubbard-Stratonovich transformation is used on $\left(\sum_{l, a_{l}, t}\right)^{2}, \sum_{l}\left(\sum_{a_{l}, t}\right)^{2}$, and $\sum_{l, a_{l}}\left(\sum_{t}\right)^{2}$. In the limit of $M \rightarrow \infty$, Eqs. (A22) and (A23) hold. By performing the Gaussian integrations over $v_{1}, v_{2}, v_{3}$, and $v_{4}, e^{G_{1}}$ can be computed as follows:

$$
\begin{aligned}
e^{G_{1}}= & \prod_{\mu} \int D v_{1} \int D v_{2} \exp \left[\frac{\beta n s}{2}(R-1)+\frac{\beta n s}{2} \frac{1+\beta_{0}}{\beta_{0}} v_{1}^{2}\right] \prod_{l} \int D v_{3} \\
& \times \prod_{a_{l}} \int D v_{4} \exp \left\{\frac{\beta s}{2}\left[v_{4} \sqrt{q_{1}-R}+i v_{1}(1-n \beta s m) \sqrt{\frac{1+\beta_{0}}{\beta_{0}}}+v_{2} \sqrt{2 m-q_{0}-n \beta s m^{2}}+v_{3} \sqrt{q_{0}-q_{1}}\right]^{2}\right\} \\
= & \prod_{\mu} \int D v_{1} \int D v_{2}\left[\frac{1}{\sqrt{1+\beta s\left(R-q_{1}\right)}}\right]^{n} \exp \left[\frac{\beta n s}{2}(R-1)+\frac{\beta n s}{2} \frac{1+\beta_{0}}{\beta_{0}} v_{1}^{2}\right] \\
& \times \prod_{l} \int D v_{3} \exp \left\{\frac{\beta m_{1} s}{2\left[1+\beta s\left(R-q_{1}\right)\right]}\left[v_{3} \sqrt{q_{0}-q_{1}}+i v_{1}(1-n \beta s m) \sqrt{\frac{1+\beta_{0}}{\beta_{0}}}+v_{2} \sqrt{2 m-q_{0}-n \beta s m^{2}}\right]^{2}\right\} \\
= & \prod_{\mu} \int D v_{1} \int D v_{2}\left[\frac{1}{\sqrt{1+\beta s\left(R-q_{1}\right)}}\right]^{n}\left[\frac{1}{\sqrt{1+\frac{\beta m_{1} s\left(q_{1}-q_{0}\right)}{1+\beta s\left(R-q_{1}\right)}}}\right]^{\frac{n}{m_{1}}} \exp \left[\frac{\beta n s}{2}(R-1)\right] \\
& \times \exp \left[\frac{\beta n s}{2}\left(\frac{1+\beta_{0}}{\beta_{0}} v_{1}^{2}+\frac{1}{\left\{1+\beta s\left[R-q_{1}+m_{1}\left(q_{1}-q_{0}\right)\right]\right\}}\left[i v_{1}(1-n \beta s m) \sqrt{\frac{1+\beta_{0}}{\beta_{0}}}+v_{2} \sqrt{2 m-q_{0}-n \beta s m^{2}}\right]\right)\right. \\
\simeq & \exp \left[\frac { \alpha n N } { 2 } \left(-\ln \left[1+\beta s\left(R-q_{1}\right)\right]-\frac{1}{m_{1}} \ln \left[1+\frac{\beta m_{1} s\left(q_{1}-q_{0}\right)}{1+\beta s\left(R-q_{1}\right)}\right]+\beta s\left\{(R-1)+\frac{1+\beta_{0}}{\beta_{0}}\right.\right.\right. \\
& \left.\left.\left.+\frac{2 m-q_{0}-\left(1+\beta_{0}^{-1}\right)}{1+\beta s\left[R-q_{1}+m_{1}\left(q_{1}-q_{0}\right)\right]}\right\}\right)\right] .
\end{aligned}
$$

We calculate $e^{G_{2}}$ under the 1RSB scheme and static approximation as follows:

$$
\begin{aligned}
e^{G_{2}}= & \sum_{\left\{\xi_{i}= \pm 1\right\}} \sum_{\left\{\tau_{i}= \pm \xi_{i}\right\}} P(\xi) P(\boldsymbol{\tau}) \operatorname{Tr}\left\{\frac{1}{2} \sinh \left[\frac{2 \beta(1-s) \lambda}{M}\right]\right\}^{\frac{n M N}{2}} \int D z \\
& \times \exp \left\{\frac{\tilde{m}}{M} \sum_{l, a_{l}, t, i} \xi_{i} \sigma_{i a_{l}}(t)+\frac{\beta(1-s)(1-\lambda)}{M} \sum_{l, a_{l}, t, i} \tau_{i} \sigma_{i a_{l}}(t)+\frac{2 \tilde{R}-\tilde{q}_{1}}{2 M^{2}} \sum_{l, a_{l}, i}\left[\sum_{t=1}^{M} \sigma_{i a_{l}}(t)\right]^{2}+\frac{\tilde{q}_{1}-\tilde{q}_{0}}{2 M^{2}} \sum_{l, i}\left[\sum_{a_{l}, t} \sigma_{i a_{l}}(t)\right]^{2}\right. \\
& \left.+\frac{\sqrt{\tilde{q}_{0}}}{M} z \sum_{l, a_{l}, t, i} \sigma_{i a_{l}}(t)+\frac{1}{2} \ln \operatorname{coth}\left[\frac{\beta(1-s) \lambda}{M}\right] \sum_{l, a_{l}, t, i} \sigma_{i a_{l}}(t) \sigma_{i a_{l}}(t+1)\right\} \\
= & \prod_{i=1}^{N} \sum_{\xi_{i}= \pm 1} \sum_{\tau_{i}= \pm \xi_{i}} \frac{1}{2} P\left(\tau_{i}\right) \int D z \prod_{l=1}^{\frac{n}{m_{1}}} \int D y \prod_{a_{l}=1}^{m_{1}} \int D x \prod_{t=1}^{M} \operatorname{Tr}\left\{\frac{1}{2} \sinh \left[\frac{2 \beta(1-s) \lambda}{M}\right]\right\}^{\frac{1}{2}} \\
& \times \exp \left\{\frac{\tilde{m}}{M} \xi_{i} \sigma_{i a_{l}}(t)+\frac{\beta(1-s)(1-\lambda)}{M} \tau_{i} \sigma_{i a_{l}}(t)+\frac{\sqrt{2 \tilde{R}-\tilde{q}_{1}}}{M} x \sigma_{i a_{l}}(t)+\frac{\sqrt{\tilde{q}_{1}-\tilde{q}_{0}}}{M} y \sigma_{i a_{l}}(t)\right.
\end{aligned}
$$




$$
\begin{aligned}
& \left.+\frac{\sqrt{\tilde{q}_{0}}}{M} z \sigma_{i a_{l}}(t)+\frac{1}{2} \ln \operatorname{coth}\left[\frac{\beta(1-s) \lambda}{M}\right] \sigma_{i a_{l}}(t) \sigma_{i a_{l}}(t+1)\right\} \\
= & \prod_{i=1}^{N} \sum_{\xi_{i}= \pm 1} \sum_{\tau_{i}= \pm \xi_{i}} \frac{1}{2} P\left(\tau_{i}\right) \int D z\left(\int D y\left\{\int D x 2 \cosh \sqrt{g\left(\tau_{i}, \xi_{i}\right)^{2}+[\beta(1-s) \lambda]^{2}}\right\}^{m_{1}}\right)^{\frac{n}{m_{1}}} \\
\simeq & \prod_{i=1}^{N} \sum_{\xi_{i}= \pm 1} \frac{1}{2} \exp \left(\frac{n}{m_{1}} \int D z \sum_{\tau_{i}= \pm \xi_{i}} P\left(\tau_{i}\right) \ln \int D y\left\{\int D x 2 \cosh \sqrt{g\left(\tau_{i}, \xi_{i}\right)^{2}+[\beta(1-s) \lambda]^{2}}\right\}^{m_{1}}\right) \\
= & \exp \left[\frac{n N}{m_{1}}\left(\sum_{a= \pm 1} c_{a} \int D z \ln \int D y\left\{\int D x 2 \cosh \sqrt{g_{a}^{2}+[\beta(1-s) \lambda]^{2}}\right\}^{m_{1}}\right)\right],
\end{aligned}
$$

where

$$
\begin{gathered}
g\left(\xi_{i}, \tau_{i}\right)=\tilde{m} \xi_{i}+\beta(1-s)(1-\lambda) \tau_{i}+\sqrt{\tilde{q}_{0}} z+\sqrt{\tilde{q}_{1}-\tilde{q}_{0}} y+\sqrt{2 \tilde{R}-\tilde{q}_{1}} x, \\
g_{a}=\tilde{m}+a \beta(1-s)(1-\lambda)+\sqrt{\tilde{q}_{0}} z+\sqrt{\tilde{q}_{1}-\tilde{q}_{0}} y+\sqrt{2 \tilde{R}-\tilde{q}_{1}} x .
\end{gathered}
$$

Under the 1RSB scheme and static approximation, $e^{G_{3}}$ is expressed as

$$
e^{G_{3}}=\exp \left\{n N\left[-m \tilde{m}-R \tilde{R}-\frac{n-m_{1}}{2} q_{0} \tilde{q}_{0}-\frac{m_{1}-1}{2} q_{1} \tilde{q}_{1}+O\left(\frac{1}{M}\right)\right]\right\} .
$$

Finally, we can obtain the 1RSB free energy density of the CDMA model in ARA as follows:

$$
\begin{aligned}
-\beta f_{1 \mathrm{RSB}}= & \operatorname{extr}_{\substack{m, q_{0}, q_{1}, R \\
\tilde{m}, \tilde{q}_{0}, \tilde{q}_{1}, \tilde{R}}}\left[\frac { \alpha } { 2 } \left(-\ln \left[1+\beta s\left(R-q_{1}\right)\right]-\frac{1}{m_{1}} \ln \left[1+\frac{\beta s m_{1}\left(q_{1}-q_{0}\right)}{1+\beta s\left(R-q_{1}\right)}\right]\right.\right. \\
& \left.+\beta s\left\{(R-1)+\frac{1+\beta_{0}}{\beta_{0}}+\frac{2 m-q_{0}-\left(1+\beta_{0}^{-1}\right)}{1+\beta s\left[R-q_{1}+m_{1}\left(q_{1}-q_{0}\right)\right]}\right\}\right) \\
& \left.-m \tilde{m}-R \tilde{R}-\frac{m_{1}-1}{2} q_{1} \tilde{q}_{1}+\frac{m_{1}}{2} q_{0} \tilde{q}_{0}+\frac{1}{m_{1}} \sum_{a= \pm 1} c_{a} \int D z \ln \int D y\left\{\int D x 2 \cosh \sqrt{g_{a}^{2}+[\beta(1-s) \lambda]^{2}}\right\}^{m_{1}}\right]
\end{aligned}
$$

The extremization condition of the 1RSB free energy density provides us with the saddle-point equations as follows:

$$
\begin{gathered}
m=\sum_{a= \pm 1} c_{a} \int D z Y_{a}^{-1} \int D y X_{a}^{m_{1}-1} \int D x\left(\frac{g_{a}}{u_{a}}\right) \sin u_{a} \\
q_{0}=\sum_{a= \pm 1} c_{a} \int D z\left[Y_{a}^{-1} \int D y X_{a}^{m_{1}-1} \int D x\left(\frac{g_{a}}{u_{a}}\right) \sin u_{a}\right]^{2} \\
q_{1}=\sum_{a= \pm 1} c_{a} \int D z Y_{a}^{-1} \int D y X_{a}^{m_{1}-2}\left[\int D x\left(\frac{g_{a}}{u_{a}}\right) \sin u_{a}\right]^{2}, \\
R=\sum_{a= \pm 1} c_{a} \int D z Y_{a}^{-1} \int D y X_{a}^{m_{1}-1} \int D x\left(\left\{\frac{[\beta(1-s) \lambda]^{2}}{u_{a}^{3}}\right\} \sinh u_{a}+\left(\frac{g_{a}}{u_{a}}\right)^{2} \cosh u_{a}\right), \\
\tilde{m}=\frac{\alpha \beta s}{1+\beta s\left(R-q_{1}\right)}, \\
\tilde{q}_{0}=\frac{\alpha \beta^{2} s^{2}\left(1+\beta_{0}^{-1}+q_{0}-2 m\right)}{\left\{1+\beta s\left[R-q_{1}+m_{1}\left(q_{1}-q_{0}\right)\right]\right\}^{2}}, \\
\tilde{q}_{1}=\tilde{q}_{0}+\frac{\alpha \beta^{2} s^{2}\left(q_{1}-q_{0}\right)}{\left\{+\beta s\left[R-q_{1}+m_{1}\left(q_{1}-q_{0}\right)\right]\right\}\left[1+\beta s\left(R-q_{1}\right)\right]}, \\
2 \tilde{R}-\tilde{q}_{1}=\frac{\alpha \beta^{2} s^{2}\left(R-q_{1}\right)}{1+\beta s\left(R-q_{1}\right)}, \\
Y_{a}=\int D y\left(X_{a}\right)^{m_{1}},
\end{gathered}
$$




$$
\begin{gathered}
X_{a}=\int D x \cosh u_{a}, \\
u_{a}=\sqrt{g_{a}^{2}+[\beta(1-s) \lambda]^{2}} .
\end{gathered}
$$

When we set $q_{0}=q_{1}=q$ and $\tilde{q}_{0}=\tilde{q}_{1}=\tilde{q}$, the 1 RSB solutions are reduced to the RS solutions. The stability of the RS solutions is evaluated by the stability analysis of the 1 RSB solutions of $q_{0}=q_{1}=q$ and $\tilde{q}_{0}=\tilde{q}_{1}=\tilde{q}$ [78]. We introduce $\Delta=q_{1}-q_{0}$ and $\tilde{\Delta}=\tilde{q}_{1}-\tilde{q}_{0}$ and take Taylor expansion to them as follows:

$$
\begin{aligned}
& \Delta=\left(\sum_{a \pm 1} c_{a} \int D z\left[\left[Y_{a}^{-1} \int D y\left(\frac{g_{a}}{u_{a}}\right) \sinh u_{a}\right]^{2}-Y_{a}^{-1} \int D y\left(\left\{\frac{[\beta(1-s) \lambda]^{2}}{u_{a}^{3}}\right\} \sinh u_{a}+\left(\frac{g_{a}}{u_{a}}\right)^{2} \cosh u_{a}\right)\right]^{2}\right) \tilde{\Delta}+O\left(\tilde{\Delta}^{2}\right), \\
& \left.\tilde{\Delta} \simeq \frac{\partial \tilde{\Delta}}{\partial \Delta}\right|_{q_{0}=q_{1}=q} \Delta+O\left(\Delta^{2}\right) \\
& \simeq \frac{\alpha \beta^{2} s^{2}}{[1+\beta s(R-q)]^{2}} \Delta \text {. }
\end{aligned}
$$

We substitute Eq. (C21) for Eq. (C22). Finally, we can obtain the stability condition:

$$
\begin{aligned}
& \frac{\alpha \beta^{2} s^{2}}{[1+\beta s(R-q)]^{2}} \sum_{a \pm 1} c_{a} \int D z\left[\left[Y_{a}^{-1} \int D y\left(\frac{g_{a}}{u_{a}}\right) \sinh u_{a}\right]^{2}-Y_{a}^{-1}\left(\int D y\left\{\frac{[\beta(1-s) \lambda]^{2}}{u_{a}^{3}}\right\} \sinh u_{a}+\int D y\left(\frac{g_{a}}{u_{a}}\right)^{2} \cosh u_{a}\right)\right]^{2} \\
& \quad<1 .
\end{aligned}
$$

[1] S. Verdu, Multiuser Detection, 1st ed. (Cambridge University Press, Cambridge, 1998).

[2] H. Nishimori, Statistical Physics of Spin Glasses and Information Processing: An Introduction (Oxford University Press, Oxford, New York, 2001).

[3] T. Tanaka, Statistical mechanics of CDMA multiuser demodulation, Europhys. Lett. 54, 540 (2001).

[4] T. Tanaka, A statistical-mechanics approach to large-system analysis of CDMA multiuser detectors, IEEE Trans. Inf. Theory 48, 2888 (2002).

[5] M. Yoshida, T. Uezu, T. Tanaka, and M. Okada, Statistical mechanical study of code-division multiple-access multiuser detectors-analysis of replica symmetric and one-step replica symmetry breaking solutions, J. Phys. Soc. Jpn. 76, 054003 (2007).

[6] D. L. Donoho, Compressed sensing, IEEE Trans. Inf. Theory 52, 1289 (2006).

[7] D. Donoho and J. Tanner, Observed universality of phase transitions in high-dimensional geometry, with implications for modern data analysis and signal processing, Philos. Trans. R. Soc. A 367, 4273 (2009).

[8] Y. Kabashima, T. Wadayama, and T. Tanaka, A typical reconstruction limit for compressed sensing based on $L_{p}$-norm minimization, J. Stat. Mech.: Theory Exp. (2009) L09003.

[9] S. Ganguli and H. Sompolinsky, Statistical Mechanics of Compressed Sensing, Phys. Rev. Lett. 104, 188701 (2010).

[10] J.-i. Inoue, Application of the quantum spin glass theory to image restoration, Phys. Rev. E 63, 046114 (2001).

[11] Y. Otsubo, J.-i. Inoue, K. Nagata, and M. Okada, Effect of quantum fluctuation in error-correcting codes, Phys. Rev. E 86, 051138 (2012).
[12] Y. Otsubo, J.-i. Inoue, K. Nagata, and M. Okada, Code-division multiple-access multiuser demodulator by using quantum fluctuations, Phys. Rev. E 90, 012126 (2014).

[13] T. Kadowaki and H. Nishimori, Quantum annealing in the transverse Ising model, Phys. Rev. E 58, 5355 (1998).

[14] G. E. Santoro, R. Martoňák, E. Tosatti, and R. Car, Theory of quantum annealing of an Ising spin glass, Science 295, 2427 (2002).

[15] G. E. Santoro and E. Tosatti, Optimization using quantum mechanics: quantum annealing through adiabatic evolution, J. Phys. A: Math. Gen. 39, R393 (2006)

[16] A. Das and B. K. Chakrabarti, Colloquium: Quantum annealing and analog quantum computation, Rev. Mod. Phys. 80, 1061 (2008).

[17] S. Morita and H. Nishimori, Mathematical foundation of quantum annealing, J. Math. Phys. 49, 125210 (2008).

[18] R. D. Somma, D. Nagaj, and M. Kieferová, Quantum Speedup by Quantum Annealing, Phys. Rev. Lett. 109, 050501 (2012).

[19] E. Farhi, J. Goldstone, S. Gutmann, J. Lapan, A. Lundgren, and D. Preda, A quantum adiabatic evolution algorithm applied to random instances of an NP-complete problem, Science 292, 472 (2001).

[20] T. Albash and D. A. Lidar, Adiabatic quantum computation, Rev. Mod. Phys. 90, 015002 (2018).

[21] V. S. Denchev, S. Boixo, S. V. Isakov, N. Ding, R. Babbush, V. Smelyanskiy, J. Martinis, and H. Neven, What is the Computational Value of Finite-Range Tunneling? Phys. Rev. X 6, 031015 (2016).

[22] T. Albash and D. A. Lidar, Demonstration of a Scaling Advantage for a Quantum Annealer over Simulated Annealing, Phys. Rev. X 8, 031016 (2018). 
[23] S. Kirkpatrick, C. D. Gelatt, and M. P. Vecchi, Optimization by simulated annealing, Science 220, 671 (1983).

[24] M. W. Johnson, P. Bunyk, F. Maibaum, E. Tolkacheva, A. J. Berkley, E. M. Chapple, R. Harris, J. Johansson, T. Lanting, I. Perminov, E. Ladizinsky, T. Oh, and G. Rose, A scalable control system for a superconducting adiabatic quantum optimization processor, Supercond. Sci. Technol. 23, 065004 (2010).

[25] A. J. Berkley, M. W. Johnson, P. Bunyk, R. Harris, J. Johansson, T. Lanting, E. Ladizinsky, E. Tolkacheva, M. H. S. Amin, and G. Rose, A scalable readout system for a superconducting adiabatic quantum optimization system, Supercond. Sci. Technol. 23, 105014 (2010).

[26] R. Harris et al., Experimental investigation of an eight-qubit unit cell in a superconducting optimization processor, Phys. Rev. B 82, 024511 (2010).

[27] S. Boixo, T. F. Rønnow, S. V. Isakov, Z. Wang, D. Wecker, D. A. Lidar, J. M. Martinis, and M. Troyer, Evidence for quantum annealing with more than one hundred qubits, Nat. Phys. 10, 218 (2014)

[28] H. G. Katzgraber, F. Hamze, and R. S. Andrist, Glassy Chimeras Could be Blind to Quantum Speedup: Designing better Benchmarks for Quantum Annealing Machines, Phys. Rev. X 4, 021008 (2014).

[29] G. Rosenberg, P. Haghnegahdar, P. Goddard, P. Carr, K. Wu, and M. L. de Prado, Solving the optimal trading trajectory problem using a quantum annealer, IEEE J. Sel. Top. Signal Process. 10, 1053 (2016).

[30] D. Venturelli and A. Kondratyev, Reverse quantum annealing approach to portfolio optimization problems, Quantum Mach. Intell. 1, 17 (2019).

[31] F. Neukart, G. Compostella, C. Seidel, D. von Dollen, S. Yarkoni, and B. Parney, Traffic flow optimization using a quantum annealer, Front. ICT 4, 29 (2017).

[32] N. Nishimura, K. Tanahashi, K. Suganuma, M. J. Miyama, and M. Ohzeki, Item listing optimization for e-commerce websites based on diversity, Front. Comput. Sci. 1, 2 (2019).

[33] M. Ohzeki, A. Miki, M. J. Miyama, and M. Terabe, Control of automated guided vehicles without collision by quantum annealer and digital devices, Front. Comput. Sci. 1, 9 (2019).

[34] D. Crawford, A. Levit, N. Ghadermarzy, J. S. Oberoi, and P. Ronagh, Reinforcement learning using quantum Boltzmann machines, Quantum Inf. Comput. 18, 0051 (2016).

[35] M. Benedetti, J. Realpe-Gómez, R. Biswas, and A. PerdomoOrtiz, Estimation of effective temperatures in quantum annealers for sampling applications: a case study with possible applications in deep learning, Phys. Rev. A 94, 022308 (2016).

[36] F. Neukart, D. Von Dollen, C. Seidel, and G. Compostella, Quantum-enhanced reinforcement learning for finite-episode games with discrete state spaces, Front. Phys. 5, 71 (2018).

[37] M. H. Amin, E. Andriyash, J. Rolfe, B. Kulchytskyy, and R. Melko, Quantum Boltzmann Machine, Phys. Rev. X 8, 021050 (2018).

[38] A. Khoshaman, W. Vinci, B. Denis, E. Andriyash, H. Sadeghi, and M. H. Amin, Quantum variational autoencoder, Quantum Sci. Technol. 4, 014001 (2018).

[39] A. D. King et al., Observation of topological phenomena in a programmable lattice of 1,800 qubits, Nature (London) 560, 456 (2018)
[40] R. Harris et al., Phase transitions in a programmable quantum spin glass simulator, Science 361, 162 (2018).

[41] P. Weinberg, M. Tylutki, J. M. Rönkkö, J. Westerholm, J. A. Åström, P. Manninen, P. Törmä, and A. W. Sandvik, Scaling and Diabatic Effects in Quantum Annealing with a D-Wave Device, Phys. Rev. Lett. 124, 090502 (2020).

[42] K. Kitai, J. Guo, S. Ju, S. Tanaka, K. Tsuda, J. Shiomi, and R. Tamura, Designing metamaterials with quantum annealing and factorization machines, Phys. Rev. Res. 2, 013319 (2020).

[43] N. Ide, T. Asayama, H. Ueno, and M. Ohzeki, Maximum likelihood channel decoding with quantum annealing machine, in 2020 International Symposium on Information Theory and Its Applications (ISITA), Kapolei, Hawai'i (IEEE, 2020), pp. 91-95.

[44] S. Suzuki and M. Okada, Residual energies after slow quantum annealing, J. Phys. Soc. Jpn. 74, 1649 (2005).

[45] T. Jörg, F. Krzakala, J. Kurchan, and A. C. Maggs, Simple Glass Models and their Quantum Annealing, Phys. Rev. Lett. 101, 147204 (2008).

[46] A. P. Young, S. Knysh, and V. N. Smelyanskiy, First-Order Phase Transition in the Quantum Adiabatic Algorithm, Phys. Rev. Lett. 104, 020502 (2010).

[47] T. Jörg, F. Krzakala, J. Kurchan, A. C. Maggs, and J. Pujos, Energy gaps in quantum first-order mean-field-like transitions: the problems that quantum annealing cannot solve, Europhys. Lett. 89, 40004 (2010).

[48] T. Jörg, F. Krzakala, G. Semerjian, and F. Zamponi, First-Order Transitions and the Performance of Quantum Algorithms in Random Optimization Problems, Phys. Rev. Lett. 104, 207206 (2010).

[49] Y. Seki and H. Nishimori, Quantum annealing with antiferromagnetic fluctuations, Phys. Rev. E 85, 051112 (2012).

[50] Y. Seki and H. Nishimori, Quantum annealing with antiferromagnetic transverse interactions for the Hopfield model, J. Phys. A: Math. Theor. 48, 335301 (2015).

[51] H. Nishimori and K. Takada, Exponential enhancement of the efficiency of quantum annealing by non-stoquastic Hamiltonians, Front. ICT 4, 2 (2017).

[52] S. Arai, M. Ohzeki, and K. Tanaka, Dynamics of order parameters of nonstoquastic Hamiltonians in the adaptive quantum Monte Carlo method, Phys. Rev. E 99, 032120 (2019).

[53] Y. Susa, Y. Yamashiro, M. Yamamoto, and H. Nishimori, Exponential speedup of quantum annealing by inhomogeneous driving of the transverse field, J. Phys. Soc. Jpn. 87, 023002 (2018).

[54] Y. Susa, Y. Yamashiro, M. Yamamoto, I. Hen, D. A. Lidar, and H. Nishimori, Quantum annealing of the $p$-spin model under inhomogeneous transverse field driving, Phys. Rev. A 98, 042326 (2018).

[55] A. Perdomo-Ortiz, S. E. Venegas-Andraca, and A. AspuruGuzik, A study of heuristic guesses for adiabatic quantum computation, Quant. Info. Proc. 10, 33 (2011).

[56] N. Chancellor, Modernizing quantum annealing using local searches, New J. Phys. 19, 023024 (2017).

[57] M. Ohkuwa, H. Nishimori, and D. A. Lidar, Reverse annealing for the fully connected $p$-spin model, Phys. Rev. A 98, 022314 (2018).

[58] Y. Yamashiro, M. Ohkuwa, H. Nishimori, and D. A. Lidar, Dynamics of reverse annealing for the fully connected $p$-spin model, Phys. Rev. A 100, 052321 (2019). 
[59] G. Passarelli, K.-W. Yip, D. A. Lidar, H. Nishimori, and P. Lucignano, Reverse quantum annealing of the $p$-spin model with relaxation, Phys. Rev. A 101, 022331 (2020).

[60] Originally, the ARA is applied when we search the ground state in the target Hamiltonian. The search of the ground state corresponds to the maximum a posteriori (MAP) estimation. The main problem of the MPM estimation is not to search the ground state but to sample the low energy state from the Gibbs-Boltzmann distribution. Strictly speaking, we should not utilize the term "annealing" because we do not perform that in this paper. Since the MPM estimation in the zero-temperature limit corresponds to the MAP estimation, we utilize the term "ARA.".

[61] M. H. Amin, Searching for quantum speedup in quasistatic quantum annealers, Phys. Rev. A 92, 052323 (2015).

[62] N. Chancellor, S. Szoke, W. Vinci, G. Aeppli, and P. A. Warburton, Maximum-entropy inference with a programmable annealer, Sci. Rep. 6, 22318 (2016).

[63] H. Nishimori, Optimum decoding temperature for errorcorrecting codes, J. Phys. Soc. Jpn. 62, 2973 (1993).

[64] M. Suzuki, Generalized Trotter's formula and systematic approximants of exponential operators and inner derivations with applications to many-body problems, Commun. Math. Phys. 51, 183 (1976).

[65] D. Sherrington and S. Kirkpatrick, Solvable Model of a SpinGlass, Phys. Rev. Lett. 35, 1792 (1975).

[66] M. Yoshida and T. Tanaka, Analysis of sparsely-spread CDMA via statistical mechanics, in 2006 IEEE International Symposium on Information Theory, Washington (IEEE, 2006), pp. 2378-2382.

[67] We verify the dependence of the order parameters on the Trotter number in our simulations. As we increase the Trotter number, the deviation of the correlation between the Trotter slices from the RS solutions decreases. The qualitative results are like those in the magnetization.
[68] M. Opper and D. Saad (eds.), Advanced Mean Field Methods: Theory and Practice, Neural Information Processing (MIT, 2001).

[69] Y. Kabashima, A CDMA multiuser detection algorithm on the basis of belief propagation, J. Phys. A: Math. Gen. 36, 1111 (2003).

[70] D. L. Donoho, A. Maleki, and A. Montanari, Message-passing algorithms for compressed sensing, Proc. Natl. Acad. Sci. USA 106, 18914 (2009)

[71] https://github.com/OpenJij/OpenJij.

[72] S. Franz and G. Parisi, Recipes for metastable states in spin glasses, J. Phys. I (France) 5, 1401 (1995).

[73] S. Franz and G. Parisi, Phase Diagram of Coupled Glassy Systems: A Mean-Field Study, Phys. Rev. Lett. 79, 2486 (1997).

[74] S. Franz and G. Parisi, Effective potential in glassy systems: Theory and simulations, Physica A 261, 317 (1998).

[75] H. Huang, K. Y. M. Wong, and Y. Kabashima, Entropy landscape of solutions in the binary perceptron problem, J. Phys. A: Math. Theor. 46, 375002 (2013).

[76] H. Huang and Y. Kabashima, Origin of the computational hardness for learning with binary synapses, Phys. Rev. E 90, 052813 (2014).

[77] J. R. L. de Almeida and D. J. Thouless, Stability of the Sherrington-Kirkpatrick solution of a spin glass model, J. Phys. A: Math. Gen. 11, 983 (1978).

[78] A. Sakata and Y. Xu, Approximate message passing for nonconvex sparse regularization with stability and asymptotic analysis, J. Stat. Mech.: Theory Exp. (2018) 033404.

[79] W. Krauth and M. Mézard, Storage capacity of memory networks with binary couplings, J. Phys. France 50, 3057 (1989).

[80] T. Obuchi, H. Nishimori, and D. Sherrington, Phase diagram of the $p$-spin-interacting spin glass with ferromagnetic bias and a transverse field in the infinite- $p$ limit, J. Phys. Soc. Jpn. 76, 054002 (2007). 